San Jose State University

SJSU ScholarWorks

Master's Projects

Master's Theses and Graduate Research

Spring 5-16-2019

\title{
Toward On-demand Profile Hidden Markov Models for Genetic Barcode Identification
}

Jessica Sheu

San Jose State University

Follow this and additional works at: https://scholarworks.sjsu.edu/etd_projects

Part of the Artificial Intelligence and Robotics Commons, and the Other Computer Sciences Commons

\section{Recommended Citation}

Sheu, Jessica, "Toward On-demand Profile Hidden Markov Models for Genetic Barcode Identification" (2019). Master's Projects. 671.

DOI: https://doi.org/10.31979/etd.qg3k-5ufh

https://scholarworks.sjsu.edu/etd_projects/671

This Master's Project is brought to you for free and open access by the Master's Theses and Graduate Research at SJSU ScholarWorks. It has been accepted for inclusion in Master's Projects by an authorized administrator of SJSU ScholarWorks. For more information, please contact scholarworks@sjsu.edu. 
Toward On-demand Profile Hidden Markov Models for Genetic Barcode Identification

\author{
A Thesis Project \\ Presented to \\ The Faculty of the Department of Computer Science \\ San José State University
}

\author{
In Partial Fulfillment \\ Of the Requirements for the Degree \\ Master of Science
}

by

Jessica Sheu

May 2019 
TOWARD ON-DEMAND PROFILE HIDDEN MARKOV MODELS FOR GENETIC BARCODE IDENTIFICATION

(C) 2019

Jessica Sheu

ALL RIGHTS RESERVED 
The Designated Project Committee Approves the Project Titled

Toward On-demand Profile Hidden Markov Models for Genetic Barcode Identification

by

Jessica Sheu

APPROVED FOR THE DEPARTMENT OF COMPUTER SCIENCE

San José State University

May 2019

Dr. Philip Heller Department of Computer Science

Dr. Sami Khuri Department of Computer Science

Dr. Wendy Lee Department of Computer Science 


\section{ABSTRACT}

Toward On-demand Profile Hidden Markov Models for Genetic Barcode Identification

By

Jessica Sheu

Genetic identification aims to solve the shortcomings of morphological identification. By using the cytochrome $c$ oxidase subunit 1 (COI) gene as the Eukaryotic "barcode," scientists hope to research species that may be morphologically ambiguous, elusive, or similarly difficult to visually identify. Current COI databases allow users to search only for existing database records. However, as the number of sequenced, potential COI genes increases, COI identification tools should ideally also be informative of novel, previously unreported sequences that may represent new species. If an unknown COI sequence does not represent a reported organism, an ideal identification tool would report taxonomic ranks to which the sequence is likely to belong. A potential solution is to dynamically create profile hidden Markov models (PHMMs): first at the genus level, then at the family level, traversing to higher taxonomic ranks until a significant score is found. This study experiments with creating PHMMs at the genus level, determining thresholds for classification, and assessing the general performance of this method and the requirements for future expansion to higher taxonomic groups. It ultimately determines that this model shows potential, but may require additional data pre-processing and may fall victim to current machine limitations.

Index Terms-Classification, cytochrome $c$ oxidase subunit 1, DNA barcoding, genetic identification, profile hidden Markov models, taxonomy. 


\section{Acknowledgements}

Throughout my two years at San José State University, I have been constantly moved and inspired by the continuous passion, ambition, and kindness of my mentors and peers. My experience as a graduate student here has been extremely fulfilling and inspiring (and often very, very challenging!), and I thank all of those who helped me decide to apply to this program, who have supported me throughout, and who have accompanied me throughout this journey. In particular, I would like to thank my family, Daniel, and Penny for their endless patience, encouragement, and love.

I would like to thank Dr. Sami Khuri for not only being the mentor of my advisor and other committee member, but also of mine and of anyone else who simply shows the wonder and motivation to learn. I will never forget the stories he told me of when he taught abroad: of young people who do not have the privileges of higher education that many of us do, and who therefore make sure to take advantage of all available educational opportunities that they can - even if it requires them to travel for hours to get there. These stories remain in my mind as strong examples of the resilience of the curious, and as reminders to practice gratitude as often as I can.

My sincerest gratitude to Dr. Wendy Lee, who, with her compassion, enthusiasm, and thorough background in Computer Science, Mathematics, Molecular Biology, Microbiology, and Bioinformatics - while also as a great teacher for SJSU students and mother to her kids - has been a great role model and shining example of how it really is possible for us to "do it all."

Finally, I would like to try to express my gratitude to Dr. Philip Heller, who has been my advisor from (literally) day one. From the first day of my first semester, he has inspired me with what I can only describe as his genuine zest for life. He has continuously shown that his drive to better the future applies not only to the macro level (the environment, the ocean, Earth), but also the micro level - in particular, the lives of us as students and members of the future generation. He has regularly made sure to consciously anticipate his students' states of mind, always intercepting any creeping pressures or negativity before they overpower us, and making the deliberate effort to empathize with our situations without us ever needing to say anything first. In addition, his personal story has been a continuous lesson for me to never again believe that we can only follow one path, and instead that we can change course at any time as long as we stay curious and work hard. I would like to thank him for providing me with invaluable guidance and support - both academic and personal - throughout my graduate experience, for always being so willing to connect me with his network of equally great people, and for providing me with truly enriching opportunities that will define my years to come. 
Table of Contents

List of Tables $\quad$ VIII

List of Figures $\quad$ X

Chapter 1: Introduction $\quad 1$

Taxonomic Classification 1

Genetic Identification using DNA Barcoding 2

COI Databases $\quad 4$

$\begin{array}{ll}\text { Identification Tools } & 6\end{array}$

Chapter 2: Data Collection and Preparation 9

$\begin{array}{ll}\text { Original Input File } & 9\end{array}$

$\begin{array}{lr}\text { Local File Database Creation } & 10\end{array}$

Chapter 3: Implementation $\quad 12$

Taxonomic Tree Creation 12

$\begin{array}{ll}\text { Evaluation Options } & 16\end{array}$

Option 1: Genus members 16

$\begin{array}{ll}\text { Option 2: Family members } & 17\end{array}$

$\begin{array}{ll}\text { Option 3: Non-members } & 18\end{array}$

$\begin{array}{ll}\text { Determining Qualifying Families } & 20\end{array}$

Building Profile Hidden Markov Models $\quad 23$

Handling Test and Train Sets 23

$\begin{array}{ll}\text { Performing Multiple Sequence Alignment } & 23\end{array}$

Building the Profile Hidden Markov Model 23

$\begin{array}{ll}\text { Conceptual Flow } & 24\end{array}$

Chapter 5: Hardware, Protocol, and Results 26

Hardware $\quad 26$

$\begin{array}{ll}\text { Protocol } & 26\end{array}$

$\begin{array}{ll}\text { Results } & 26\end{array}$ 
$\begin{array}{ll}\text { Acanthosomatidae } & 30\end{array}$

$\begin{array}{ll}\text { Agabini } & 32\end{array}$

$\begin{array}{ll}\text { Agelenidae } & 35\end{array}$

$\begin{array}{ll}\text { Harpalini } & 38\end{array}$

$\begin{array}{ll}\text { Hydraenidae } & 40\end{array}$

$\begin{array}{ll}\text { Hydropsychinae } & 43\end{array}$

$\begin{array}{ll}\text { Ophiolepididae } & 50\end{array}$

$\begin{array}{ll}\text { Triozidae } & 52\end{array}$

Vespinae $\quad 55$

$\begin{array}{ll}\text { Precision and Recall } & 57\end{array}$

Chapter 6: Discussion $\quad 59$

$\begin{array}{ll}\text { Overall Performance } & 59\end{array}$

$\begin{array}{ll}\text { Testing on Non-Members } & 59\end{array}$

Testing on Family Members $\quad 59$

$\begin{array}{ll}\text { Testing on Genus Members } & 61\end{array}$

$\begin{array}{ll}\text { Accuracy Correlation Analysis } & 64\end{array}$

Primer Isolation Method $\quad 65$

$\begin{array}{ll}\text { Machine Limitations } & 67\end{array}$

$\begin{array}{ll}\text { Future Directions } & 68\end{array}$

Handling Varying Sequence Length and Disqualifying Outliers $\quad 70$

$\begin{array}{ll}\text { Creating Multiple PHMMs for Each Genus } & 70\end{array}$

$\begin{array}{ll}\text { References } & 72\end{array}$ 
Table I: Example Test-Train Splits for Genus with 11 Members 17

Table II: Tuned Qualifications vs. Resulting Test Set Size 19

Table III: Random Families Selected and Their Corresponding Genera 22

Table IV: General Genus Information $\quad 29$

Table V: Acanthosomatidae Acanthosoma Results Summary 30

Table VI: Acanthosomatidae Elasmucha Results Summary 31

Table VII: Agabini Agabus Results Summary 32

Table VIII: Agabini Ilybius Results Summary 33

Table IX: Agabini Platynectes Results Summary 34

Table X: Agelenidae Malthonica Results Summary 35

Table XI: Agelenidae Sinocoelotes Results Summary 36

Table XII: Agelenidae Tegenaria Results Summary 37

Table XIII: Harpalini Harpalus Results Summary 38

Table XIV: Harpalini Ophonus Results Summary 39

Table XV: Hydraenidae Hydraena Results Summary $\quad 40$

Table XVI: Hydraenidae Limnebius Results Summary $\quad 41$

Table XVII: Hydraenidae Ochthebius Results Summary 42

Table XVIII: Hydropsychinae Ceratopsyche Results Summary 44

Table XIX: Hydropsychinae Cheumatopsyche Results Summary 45

Table XX: Hydropsychinae Hydromanicus Results Summary 46

Table XXI: Hydropsychinae Hydropsyche Results Summary 47

Table XXII: Hydropsychinae Orthopsyche Results Summary 48

Table XXIII: Hydropsychinae Potamyia Results Summary 49

Table XXIV: Ophiolepididae Ophiolepis Results Summary 50

Table XXV: Ophiolepididae Ophiomusium Results Summary

Table XXVI: Triozidae Myotrioza Results Summary 52

Table XXVII: Triozidae Pariaconus Results Summary 53

Table XXVIII: Triozidae Trioza Results Summary $\quad 54$ 
TOWARD ON-DEMAND PROFILE HIDDEN MARKOV MODELS FOR GENETIC BARCODE IDENTIFICATION

Table XXIX: Vespinae Vespa Results Summary

Table XXX: Vespinae Vespula Results Summary 56

Table XXXI: Precision and Recall for Genus Classification 58 


\section{List of Figures}

Fig. 1: Sample Taxonomic Subtree with Repeated Taxonomic Groups 15

Fig. 2: Minimum Number of Genera and Species vs. Number of Qualifying Families 22

Fig. 3: Project Conceptual Flowchart 25

Fig. 4: Acanthosomatidae Acanthosoma PHMM Results 30

Fig. 5: Acanthosomatidae Elasmucha PHMM Results 31

Fig. 6: Agabini Agabus PHMM Results 32

Fig. 7: Agabini Ilybius PHMM Results 33

Fig. 8: Agabini Platynectes PHMM Results 34

Fig. 9: Agelenidae Malthonica PHMM Results

Fig. 10: Agelenidae Sinocoelotes PHMM Results 36

Fig 11: Agelenidae Tegenaria PHMM Results $\quad 37$

Fig. 12: Harpalini Harpalus PHMM Results 38

Fig. 13: Harpalini Ophonus PHMM Results 39

Fig. 14: Hydraenidae Hydraena PHMM Results $\quad 40$

Fig. 15: Hydraenidae Limnebius PHMM Results $\quad 41$

Fig. 16: Hydraenidae Ochthebius PHMM Results 42

Fig. 17: Hydropsychinae Ceratopsyche PHMM Results 43

Fig. 18: Hydropsychinae Cheumatopsyche PHMM Results 45

Fig. 19: Hydropsychinae Hydromanicus PHMM Results 46

Fig. 20: Hydropsychinae Hydropsyche PHMM Results 47

Fig. 21: Hydropsychinae Orthopsyche PHMM Results 48

Fig. 22: Hydropsychinae Potamyia PHMM Results 49

Fig. 23: Ophiolepididae Ophiolepis PHMM Results 50

Fig. 24: Ophiolepididae Ophiomusium PHMM Results 51

Fig. 25: Triozidae Myotrioza PHMM Results 12

Fig. 26: Triozidae Pariaconus PHMM Results 53

Fig. 27: Triozidae Trioza PHMM Results $\quad 54$

Fig. 28: Vespinae Vespa PHMM Results 
TOWARD ON-DEMAND PROFILE HIDDEN MARKOV MODELS FOR GENETIC BARCODE IDENTIFICATION

Fig. 29: Vespinae Vespula PHMM Results 


\section{Chapter 1: Introduction}

\section{Taxonomic Classification}

The earliest records of taxonomic classification were by Aristotle (384-322 B.C.) [1]. In its earliest form, animal taxonomy was based on Aristotle's personal logic and inspection; he classified animals based on whether they were egg-bearing or not, four-legged or not, landbearers or water-bearers, "with blood" or "bloodless," and so on [1]. Modern taxonomy was founded more than a millennium later by the "father of taxonomy," Carl Linnaeus (17071778) [1]. Linnaeus introduced binomial nomenclature, or the scientific naming of organisms using two parts: their genus name followed by their species name [1]. For example, the scientific name of the human is Homo sapiens, where Homo is the genus name and sapiens is the species name. In addition, he adopted a four-level hierarchy: class, order, genus and species [1]. This is similar to the hierarchy primarily used today, which consists of: domain, kingdom, phylum, class, order, family, genus, and species. For further classification, there exist subcategories such as subphylum, subclass, suborder, and so on.

When scientists today study organisms or field samples, it is often unreliable for them to identify their findings through morphological, or physical, traits as Aristotle and Linnaeus did in the past [1]. For example, scientists are interested in differentiating invasive species from native species to determine their effect on the community. This research can also scrutinize the rate of growth of the invasive colonies. However, invasive species can be physically identical to the native species, making it highly improbable for even a highly 
trained scientist to accurately visually identify one species from the other when encountered in the wild [2] [3]. Conversely, other scientists are interested in studying only a particular species, but even individual organisms within species can be morphologically different due to their sex or age [4]. Furthermore, scientists are interested in taking environmental samples to study the contained microbial communities and non-living contents [4]. This research can further inform them of the genetic potential of the areas in which the samples were taken. However, organisms in sampled microbial communities are too small and too high in number for visual identification [5]. From these few examples, it is evident that morphologically identifying organisms is neither reliable nor efficient. Genetic identification aims to solve this problem.

\section{Genetic Identification using DNA Barcoding}

It may seem that genetic identification faces the same issues as morphological identification; organisms certainly also differ from each other genetically. It was therefore imperative for scientists to find a standardized genetic "barcode" gene that was present in all organisms regardless of their species, and whose sequence was unique to each species [4]. In addition, scientists wanted this gene to be minimum length (ideally 150 base pairs or fewer) so that it could be easily amplified, or duplicated [4] [6]. The current proposed gene for animals is the mitochondrial cytochrome $c$ oxidase subunit 1 (COI) gene [4]. This 658 base pair sequence is present in all organisms of the animal kingdom and was found to be highly informative even within organisms of the same order [6]. Other than its length, the COI gene meets all other criteria, and a better suited gene has not yet been discovered [4]. 
Although DNA barcoding has been described by some groups as "anti-taxonomy" [6], it has many potential and current advantageous applications. The DNA barcode can be extracted from small traces of animals such as their hair, feces, or urine. This allows ecologists to study species that are otherwise elusive [4]. In addition, DNA barcoding can distinguish among cryptic species: organisms that are morphologically identical but that belong to different species. For example, the butterfly Astraptes fulgerator was believed to be a single species from its morphological traits [7]. However, using DNA barcoding, Hebert et al. found $A$. fulgerator is actually a group of at least ten separate, but morphologically identical, species [7]. Evidently, genetic identification can overcome limitations of morphological identification.

Not only can DNA barcoding be used with live animals but also with animal byproducts and food processing [4] [8]. For example, some species of fish are preprocessed (sliced, frozen, canned, and so on) before being sold. As with morphologically identifying live species, it is also difficult to use traditional identification methods to trace the origin of the fish or determine whether the food has been mislabeled [8]. Animal byproducts, such as eggs from different fish species, are also difficult to visually identify [4]. DNA barcoding excels in this situations; since the DNA can be extracted from small traces of the animals, scientists can quickly trace the fish and discover possible illegal sales of endangered fish eggs [4] [8].

Although DNA barcoding does not necessarily always bring an exact species match, it narrows the search and therefore makes the search more efficient. Because of this, it has brought "species-level resolution" in 95\% to 97\% of trials studying different animal groups 
[9]. In addition, DNA barcoding provides answers 3-4 times more quickly than traditional taxonomic methods [10], at only $\$ 2.50$ to $\$ 8$ a sample [4]. While additional sequencing costs mean that DNA barcoding may not be more inexpensive than traditional taxonomic methods, scientists view its much quicker analysis time as very valuable and expect the pricing to decrease as technology advances [10].

When we scan an item's barcode in a supermarket, the identity of the item must be stored somewhere so that the machine can look it up. Once the machine recognizes which item is being scanned, it can then charge us accordingly. Similarly, to be able to use an organism's genetic barcode for identification, we must first have an accurate database of gathered genetic barcodes. Then, to actually identify a barcode, we must have a tool that we can use to "scan" it. This tool will then let us know what organism the barcode is associated with it (if it exists in the database).

\section{COI Databases}

An ideal COI database would have the largest number available COI sequences to-date and, of course, be highly accurate. In this section we will discuss three database options: The Barcode of Life Data System (BOLD) [11], Midori-UNIQUE [12], and, more recently, a database data-mined from GenBank using CO-ARBitrator [13].

BOLD [11] was created by the Consortium for the Barcode of Life (CBOL), an organization that aimed to develop and publicize a library containing COI barcodes for all discovered animals [11] [13]. For a COI record to be accepted to BOLD, it must be submitted with 
seven data criteria: the species name, voucher data, collection record, specimen identifier, COI sequence, polymerase chain reaction (PCR) primers, and trace files [11]. Because of these stringent requirements, entry into BOLD is slow. In addition, gathering the data elements needed for consideration is expensive. There are therefore fewer COI records in BOLD than are available on the GenBank database; as such, BOLD does not have the largest number of available COI sequences. However, because of its entry criteria, the sequences in BOLD are likely to be more accurate.

In contrast, the COI records in the Midori-UNIQUE [12] database are acquired from GenBank mostly based on their annotations. Although this provides the benefit of quickly growing publicly-available data that combats the slow entry into BOLD, the sequences uploaded to GenBank can be misannotated due to misspellings or misidentifications from human error [13] [14]. Because of this, the Midori-UNIQUE database is less accurate than BOLD. Furthermore, despite Midori-UNIQUE's more lenient criteria, it also still does not contain all COI records available in GenBank.

Heller et al.'s [13] [15] research acknowledges the usefulness of GenBank and its extremely large amount of available sequences. They also recognize that results from the Basic Local Alignment Search Tool (BLAST) [16] can accurately provide sequences of one's original gene query. However, Heller et al. found that BLAST output may also contain false positives. They therefore propose that an effective database curation algorithm must not only use BLAST, but must have subsequent steps to optimize the BLAST output. The algorithm should take the plentiful output of BLAST and change it from being overly 
sensitive to specific. In other words, the algorithm should take the set containing sequences of the target gene along with false positives, then output a final set containing sequences of only the target gene.

Heller et al. expanded CO-ARBitrator [13] from their original software, ARBitrator [15], in order to work with animal COI genes. CO-ARBitrator evaluates sequences on thresholds of sensitivity and specificity that are specific to the target gene and must be calculated. As a result, CO-ARBitrator accepted 1,054,973 COI sequences, of which approximately half were not in BOLD and approximately half were not in the Midori-UNIQUE database [13]. In addition, its false positive rate was calculated to be less than $0.0034 \%$ [13].

By filtering out the undesired BLAST output contents, CO-ARBitrator provides a much higher sequence extraction accuracy when compared to BLAST, and its acquired sequence database contained approximately 500,000 more records than BOLD and Midori-UNIQUE. We will therefore be using the CO-ARBitrator COI database for this project.

\section{Identification Tools}

An ideal identification tool would be quick, robust, and accurate. Current COI archives allow users to search for already reported sequences; however, there is a need for an identification tool that can also be informative with unknown sequences. Given a novel sequence that is previously unreported and presumably from a newly discovered species, this tool would be able to determine the most probable genus of the sequence using our highly-populated COI database. If the tool is not able to determine the most probable genus, 
we hope that it could report another possible taxonomic rank to which the sequence is likely to belong.

Sharma's [17] work explores the application of profile hidden Markov models (PHMMs) for genetic identification. Given an unknown COI sequence, PHMMs are created at the genus level. The algorithm checks whether a sequence receives a significant score when tested against its own genus. Sharma tested this method of genetic identification by creating PHMMs at the genus level using 106 total sequences across 12 phyla: Annelida, Arthropoda, Bryozoa, Chordata, Cnidaria, Echinodermata, Mollusca, Nematoda, Platyhelminthes, Porifera, Rotifera, and Brachiopoda. He reported a total error rate across all phyla to be 9.43\%, and notes that the highest number of errors were found in the phyla Arthropoda and Rotifera. For the other phyla, he reported the error rate to be $0.9 \%$.

In the future, this method can be expanded to continue to dynamically create PHMMs at higher taxonomic levels until a conclusion about the sequence can be made. If no significant scores are found at the genus level, the future program will create PHMMs for the next taxonomic rank, at the family level. The program will continue to create PHMMs at each higher level while no significant scores are found. As an example, if there is no significant score with a particular species at the genus level but there is a good score for one genus at the family level, this will be an indication that we may have found a new species within that genus. To be clear, the program will not guarantee that a new species has been found, but the results will indicate that this is an area to investigate. On-demand PHMM creation has potential to be hugely advantageous to morphological identification: not only will it save 
memory and time, but users will also not need to be highly trained taxonomists to interpret the results.

This project will be a continuation of Sharma's [17] conceptual work; however, it will involve original implementation and the COI dataset acquired by CO-ARBitrator [13] instead of BOLD. In this study, we will create PHMMs only at the genus level to determine the adjustments and measures needed for future expansion to higher taxonomic ranks. We plan on testing multiple families that consist of multiple sizeable genera. With this data we will be able to not only observe the results of each individual genus, but also compare accuracy across family members. We plan on assessing the potential of this classification method and hope to see a positive correlation between genus size and accuracy. In other words, we hope that this project will produce results that will show that, if more data is to be acquired and added to the COI database, accuracy of our classification system will improve. 


\section{Chapter 2: Data Collection and Preparation}

This chapter describes the input file used for this project and the format of the contained records. It further details our implementation to create a local database that allowed us to access the lengthier COI sequences with the shorter accession numbers stored in RAM.

\section{Original Input File}

This project used an input fasta file of 863,757 total COI sequences gathered by COARBitrator [13]. Each record in this file consisted of a defline on one line and the corresponding genetic sequence on the next. In this file, an organism was typically represented with a defline that followed the format:

>AccessionNumber_Genus;Species;Eukaryota;Phylum;Class;Order;Family;Genus

Although the example defline above only contains the main taxonomic groups, it should be noted that a typical defline in this input file contained additional groups, such as subphylum, subclass, and so on. Each defline could contain a different number of taxonomic groups; therefore, the format of the deflines were not uniform. However, in general the defline did follow the format:

>AccessionNumber_Genus;Species;Eukaryota;RemainingTaxonomicGroupsUntilGenus 


\section{Local File Database Creation}

It is undesirable to store 1 million sequences that are each approximately 300-900 base pairs in length in RAM on a typical machine. This project's better alternative was to store the much shorter accession numbers ( 6 to 12 characters each) in RAM and use them as keys to look up the corresponding genetic sequence.

It is also undesirable to look through this original input file; a linear search of almost 1 million sequences each time the program tries to access a single sequence is inefficient and unnecessary. Therefore, this project built a local database to improve on lookup time given an accession number string.

This feature allowed the user to specify the number of levels they would like in their database. The database creating method then used that number of characters in the accession number. Each character from 0 to numLevels- 2 was used to create a directory. Each directory was a contained subdirectory of the directory created before it. The last character at position numLevels- 1 in the accession number was used as the name of the fasta file that would then contain this accession number and sequence.

For example, given an accession number AB01234, if the user specified the number of levels to be 3:

Parent directory (first character): A/

Contained subdirectory (second character): B/

Contained .fasta file (third character): $0 . \mathrm{fa}$ 
Full path: $A / B / 0 . f a$

This file would contain all records with an accession number of the following format: AB0<any_other_characters >. In total, there would be 3 levels: 2 directories and 1 file.

To look up a sequence given an accession number, the program searched for a parent directory with the name of the first character of the accession number, a contained subdirectory with the name of the second character, and a contained fasta file with the name of the third character. It then looked through all entries of the fasta file to find a defline that contained the accession number query. If found, the method returned the sequence, which was in the next line. If the accession number could not be found in the file, the method returned null. While the lookup search for this method was still linear, this local database creation greatly reduced the number of lines to search through in each file while also allowing us to store only the accession numbers in RAM. 


\section{Chapter 3: Implementation}

This chapter details our implementation of our proposed identification tool, the three included test features and their purposes, the experiments that led us to select our training and test sets, and the additional software used. It ends with a conceptual flow diagram that visually summarizes all features described in this chapter.

\section{Taxonomic Tree Creation}

A Java class was created to store the taxonomic levels of all organisms in the fasta input file. Because COI is the "barcode" gene for Eukaryotes, all organisms within the data set are in the shared domain Eukaryota; this group name was set as the root node of the tree.

This class parsed each defline of the input file. For each iteration, the current node was initialized to the root node: Eukaryota. At each taxonomic group, the class checked if this group already existed as a child of the current node. If not, the method created a new child with the current group name. If this group name was at species level, this child node was also initialized to store the accession number. This is because only nodes at the species level can be represented by a single accession number. In any case, the current node was set as the node with the current group name.

Although the input file contained multiple records for some species, the program retained only a single record for each species. In addition, any records with deflines that contained a $\because$ character (such as $s p$. or $c f$.) or the string "environmental;sample" were not included in 
the tree. This is because records listed as "sp." (species), "cf." (confer/compare to), or "environmental sample" do not yet represent known species. It is possible that there are multiple records that are labeled "sp." (for example) in the same genus, but these would likely all represent different organisms. Despite this, due to the method we used to build the tree, additional organisms labeled as "sp." would be forgone if a child node with that group name already existed. Furthermore, it would not be useful if our identification tool classified an unknown sequence with these ambiguous labels.

The program proceeded with the remainder of the input file. It should be noted that each defline from the input file lists a varying number of taxonomic groups. An example of two deflines from the input dataset is shown below:

>AB021146_Orius;sauteri_Eukaryota;Metazoa;Ecdysozoa;Arthropoda;

Hexapoda;Insecta;Pterygota;Neoptera;Paraneoptera;Hemiptera;Heteroptera; Panheteroptera;Cimicomorpha;Anthocoridae;Anthocorinae;Orius

>AB021147_Orius;nagaii_Eukaryota;Arthropoda;Insecta;Hemiptera;Anthocoridae; Orius;Oriusnagaii

It can be seen that these two organisms are in the same genus (Orius). They therefore share all taxonomic groups higher than genus: kingdom (Metazoa), superphylum (Ecdysozoa), phylum (Arthropoda), and so on. Despite this, the second record does not list many of the groups that are listed in the first. The groups that are included in the first record but not in the second are shown in bold above. 
We did not make adjustments to account for these varying number of listed taxonomic groups. The produced taxonomic tree was an unbalanced, non-binary tree and had nodes on different levels with the same group name.

The subtree represented by the two deflines above are shown in Fig. 1. 


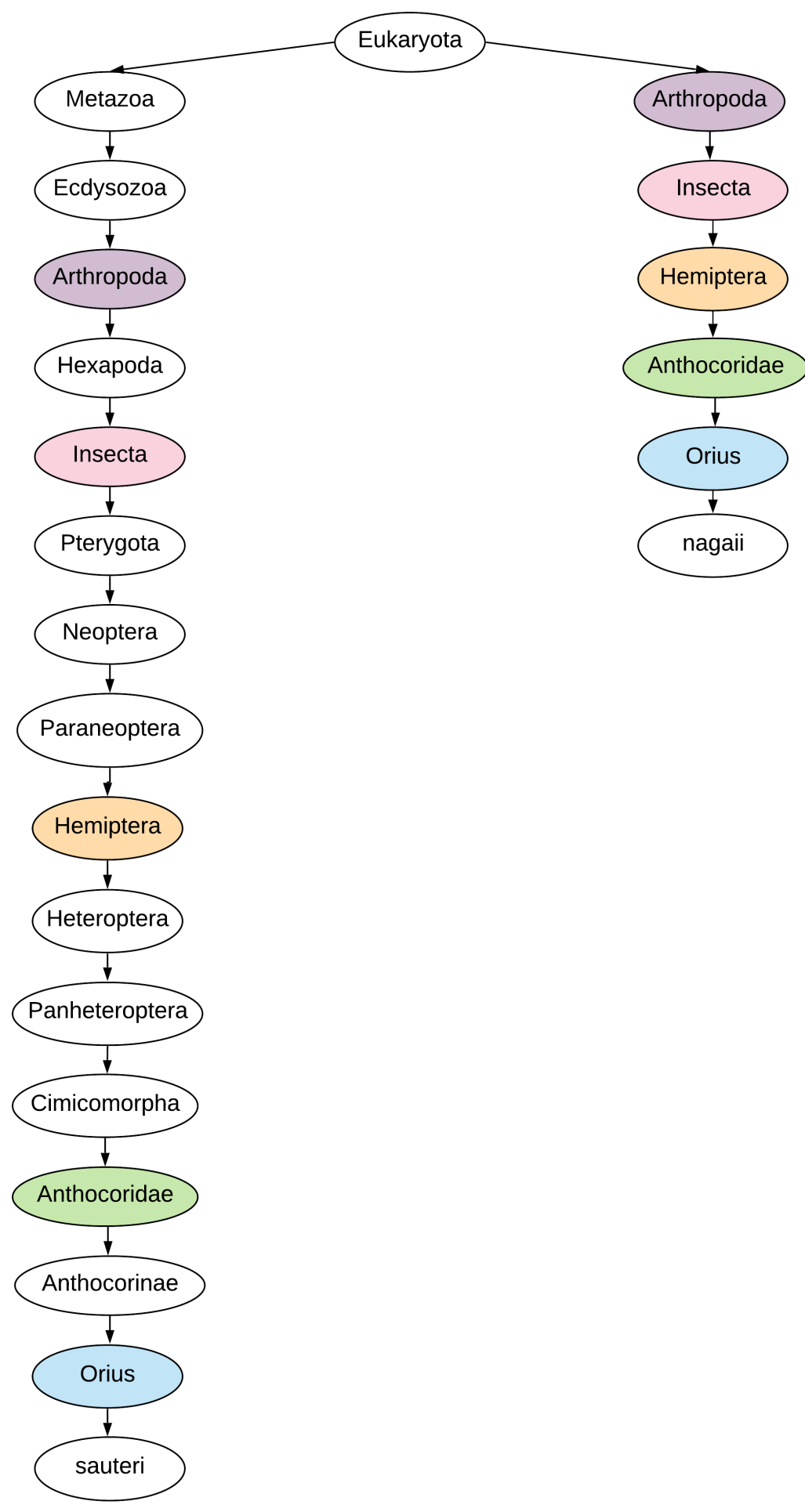

Fig. 1. Taxonomic subtree produced by the deflines with the accession numbers AB021146 and AB021147. Repeated taxonomic groups that represent the same group but are duplicated in the tree on different levels, are indicated by the same color. 


\section{Evaluation Options}

This project handled 3 possible options for training and testing a profile hidden Markov model (PHMM). Each option simulated the discovery of an unknown sequence and will lead us to determining whether we would be able to classify it based on: a typical score of a genus member against its own genus, a typical score of a family member against a genus in its family, and a typical score of a complete non-member against a genus in a family not in its own taxonomic groups. These options and their purposes are detailed below.

\section{Option 1: Genus members}

In this option, the program simulated finding an unknown sequence of a genus and testing it against the genus to which it is suspected to belong. Given a genus of size n, we retained as many members as possible to train the PHMM: $n-1$ sequences. We withheld 1 sequence to treat as an unknown sequence, use for testing, and calculate its log-odds score against the "withholding" genus PHMM.

We continued to "shuffle" single sequences in the genus for every combination of n-1 sequences for training and 1 sequence for testing. For example, Table I shows a few iterations of test-train splits for if there are 11 members of the genus. 
TABLE I

EXAMPLE TEST-TRAIN SPLITS FOR GENUS WITH 11 MEMBERS

\begin{tabular}{|c|c|c|}
\hline Iteration & Training set sequences & Test set sequence \\
\hline $\mathbf{0}$ & $0-9$ & 10 \\
\hline $\mathbf{2}$ & $1-10$ & 0 \\
\hline $\mathbf{3}$ & $0,2-10$ & 1 \\
\hline$\ldots$ & $0-1,3-10$ & 2 \\
\hline $\mathbf{9}$ & $\ldots$ & $\cdots$ \\
\hline
\end{tabular}

We received the log-odds score of the test sequence against the withholding genus PHMM at each iteration. After each genus had been completely evaluated, we recorded its threshold: the lowest log-odds score possible from a member of the genus that was not used in the training set. With this information, we could know that a typical member of the genus scores higher than this minimum score; therefore, a non-member of the genus should score below it. By testing other random sequences against this genus PHMM, we planned on making assumptions about whether or not they might belong to the genus, based on whether or not they fell above or below the minimum score threshold.

\section{Option 2: Family members}

With this option we simulated finding an unknown sequence and speculating that it belongs to a certain family, without having a strong suspicion of the specific genus. For 
each genus, the program built a non-withholding PHMM; that is, the program trained a PHMM on all members of the genus (unlike in Option \#1).

The program then built a test set on all members of the family that are not members of the genus used to train the PHMM. All family members were tested and their scores were recorded. Through these scores we could make observations on how a typical family member scores against a genus in relation to the genus members from Option \#1.

\section{Option 3: Non-members}

In this last option, we once again built a non-withholding PHMM trained on all members of a genus. The program then built a test set of records, in which the organisms were neither members of the genus nor members of the family.

We had initially attempted to test all sequences from the initial input data. However, due to machine limitations and in the interest of time and computing power, we decided to reduce the number of non-members to test for each genus PHMM. Therefore, we decided to look for the major phyla in the taxonomic tree that were of a minimum size.

Our original method was to retain only phyla that had a minimum of 1,000 species, then to take a random $10 \%$ of those phyla to create the test set. However, this did not give us many qualifying nodes - only 11 phyla had at least 1,000 species; $10 \%$ of 1,000 species for 11 phyla would give us only 1,100 test sequences. Table II shows a small selection of further experimentation with values and taxonomic group levels. 
TABLE II

TUNED QUALIFICATIONS VS. RESULTING TEST SET SIZE

\begin{tabular}{|c|c|c|c|c|}
\hline \multirow{2}{*}{$\begin{array}{c}\text { Root } \\
\text { taxonomic } \\
\text { group }\end{array}$} & $\begin{array}{c}\text { Minimum } \\
\text { number of } \\
\text { species }\end{array}$ & $\begin{array}{c}\text { Tand qualifications } \\
\text { minimum of the } \\
\text { species taken for the } \\
\text { test set }\end{array}$ & $\begin{array}{c}\text { Rumber of } \\
\text { qualifying } \\
\text { nodes }\end{array}$ & $\begin{array}{c}\text { Number of } \\
\text { resulting } \\
\text { sequences in } \\
\text { Order }\end{array}$ \\
\hline Phylum & 500 & $80 \%$ & 22 & test set \\
\hline Phylum & 550 & $100 \%$ & 17 & 8,800 \\
\hline Phylum & 600 & $100 \%$ & 15 & 8,250 \\
\hline
\end{tabular}

We decided to retain phyla that had a minimum of 600 species and take $100 \%$ of this 600 minimum species for the test set. Therefore, if a phylum had exactly 600 species, the program would write all 600 species to the test set. If a phylum had more than 600 species, the program would write a subset of 600 random species to the test set.

A question that may arise is whether taking $100 \%$ of the minimum phylum size produced a random test set. The situation where this would not produce a random test set is if the phyla had exactly 600 species. This was taken into consideration; however, the 15 qualifying phyla all had more than 600 species: they ranged from 667 to 32,000 species. The majority of these phylum nodes had more than 700 species. Therefore, we determined that this method and these chosen parameters produced a test set that was random enough for the needs of this project. 
All 9,000 sequences were written to the test set. The log-odds score for each sequence was evaluated against the non-withholding PHMM. The program attempted to evaluate as many test sequences as possible under the constraints of the machines used.

\section{Determining Qualifying Families}

Because this project involves creating profile hidden Markov models at the genus level, we were interested in finding families with multiple and sizable genera to perform crosscomparison. We experimented to determine the ideal number of genera and number of species in each genus to suit our needs for this project. Families that met these minimum requirements were considered "qualifying families."

We made the assumption that 10 species would be sufficient to train a PHMM in this project. For Evaluation Option \#1 detailed in the previous subsection, we would therefore need a minimum genus size of 11 in order to train on 10 and test on the withheld single sequence for each shuffling iteration.

We considered "families" to be nodes that were 2 levels above a leaf node. This is because the leaf nodes represent the species level so the level above represent the genus level. However, it should be noted that these nodes did not always represent the family taxonomic rank. Each organism in the input dataset was represented by a different number of taxonomic ranks. Therefore, it is possible that a node that is 2 levels above a leaf node represents an intermediate taxonomic rank, such as subgenus or subfamily. 
Because our taxonomic tree is unbalanced, it is possible that multiple nodes at the same height from a leaf node, share the same group name. For example, in the example in Fig. 1, there are two nodes with the group name Orius that are 1 level above a leaf node, but on different levels of the taxonomic tree. Therefore, we first combined families with the same group name. By doing this, we could account for all of the children of a taxonomic group, even if multiple nodes represent one group in the tree. We then checked if these families were "qualifying."

We observed the number of qualifying families returned when retaining only families with a minimum of 3 genera while varying the minimum number of species in each genus from 11 to 20 . We also experimented with a minimum of 2 genera in each family with the same range of minimum numbers of species.

Fig. 2 shows the results. There was a significant difference in number of qualifying families retained through selecting a minimum number of 2 genera when compared with a minimum number of 3 genera. We determined that experimenting with 2 genera would be sufficient for this project; we would be able to compare performance with at least 1 other member of the family, and being able to test on a much larger number of families greatly outweighed being able to test 1 additional genus. Table III shows the families that were randomly selected for this experiment, as well as their corresponding genera. 
Minimum Number of Genera and Species vs. Number of Qualifying Families

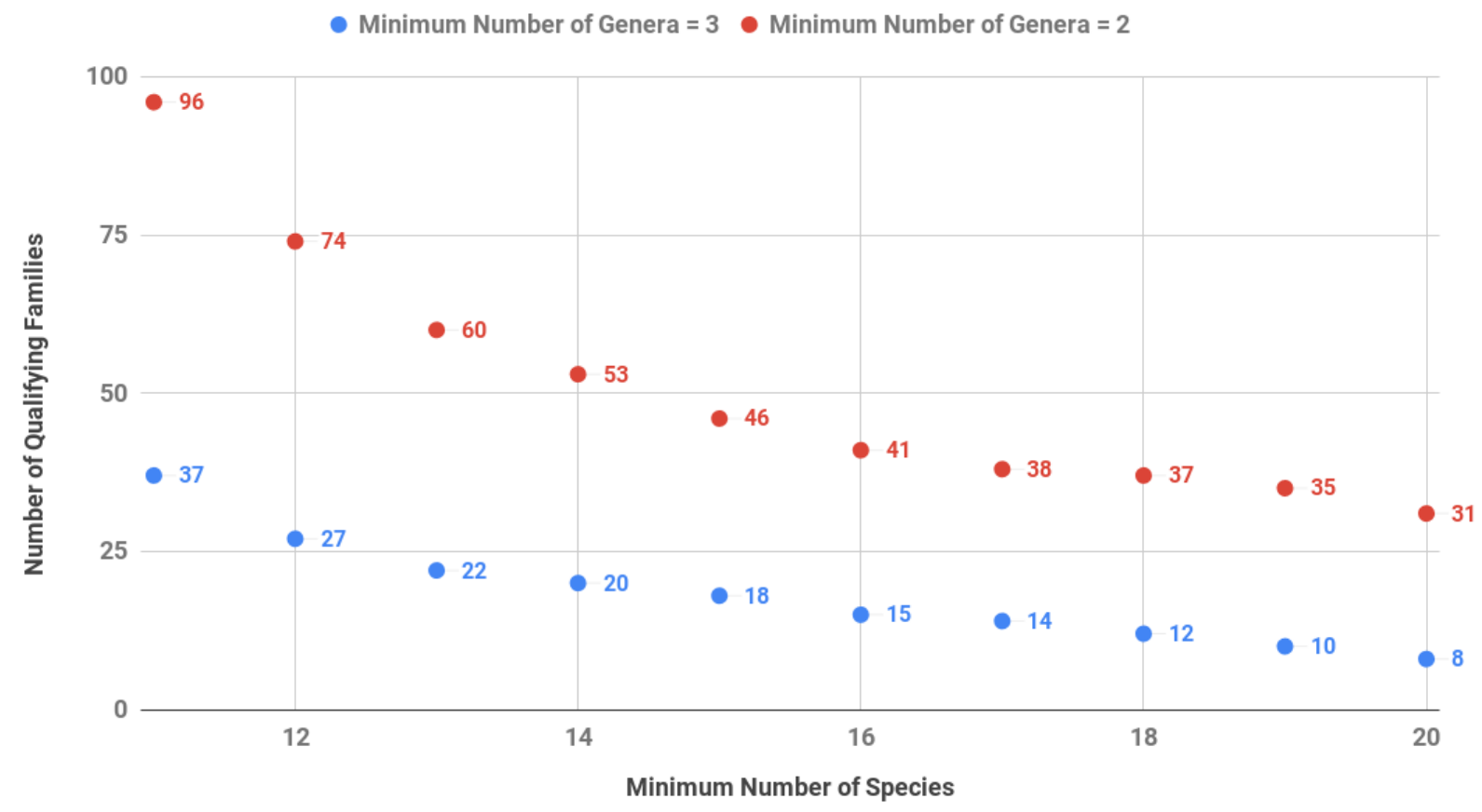

Fig. 2. Comparison of number of qualifying families resulting from varying minimum numbers of genera and species.

TABLE III

RANDOM FAMILIES SELECTED AND THEIR CORRESPONDING GENERA

\begin{tabular}{|c|c|}
\hline Family & Genera \\
\hline Acanthosomatidae & Acanthosoma, Elasmucha \\
\hline Agabini & Agabus, Ilybius \\
\hline Agelenidae & Malthonica, Sinocoelotes, Tegenaria \\
\hline Harpalini & Harpalus, Ophonus \\
\hline Hydraenidae & Hydraena, Limnebius, Ochthebius \\
\hline Hydropsychinae & Ceratopsyche, Cheumatopsyche, Hydromanicus, Hydropsyche, Orthopsyche, Potamyia \\
\hline Ophiolepididae & Ophiolepis, Ophiomusium \\
\hline Triozidae & Myotrioza, Pariaconus \\
\hline Vespinae & Vespa, Vespula \\
\hline
\end{tabular}




\section{Building Profile Hidden Markov Models}

\section{Handling Test and Train Sets}

Test and train sets were written to temporary files that were overwritten by each iteration so to not take up an unnecessarily large amount of memory.

\section{Performing Multiple Sequence Alignment}

We used Clustal Omega ${ }^{1}$ for multiple sequence alignment in preparation for building a profile hidden Markov model (PHMM). When executing this binary within our Java program, Clustal Omega takes as input the training file of sequences in .fasta format. It writes the resulting alignment to an output fasta file.

\section{Building the Profile Hidden Markov Model}

This project uses Dr. Philip Heller's Alignment and ProfileHMM classes to build a PHMM and compute a log-odds Viterbi score. The Alignment class stores the multiple sequence alignment from the output file produced by Clustal Omega. This Alignment object is then used to build the PHMM.

1 http://www.clustal.org/omega/\#Documentation 


\section{Recording Output}

All log-odds scores were written to a .csv file for each individual genus. The accession numbers of each tested organism constituted the first column of the file, with their corresponding log-odds score in the second column.

\section{Conceptual Flow}

Fig. 3 shows the conceptual flow of this project. 


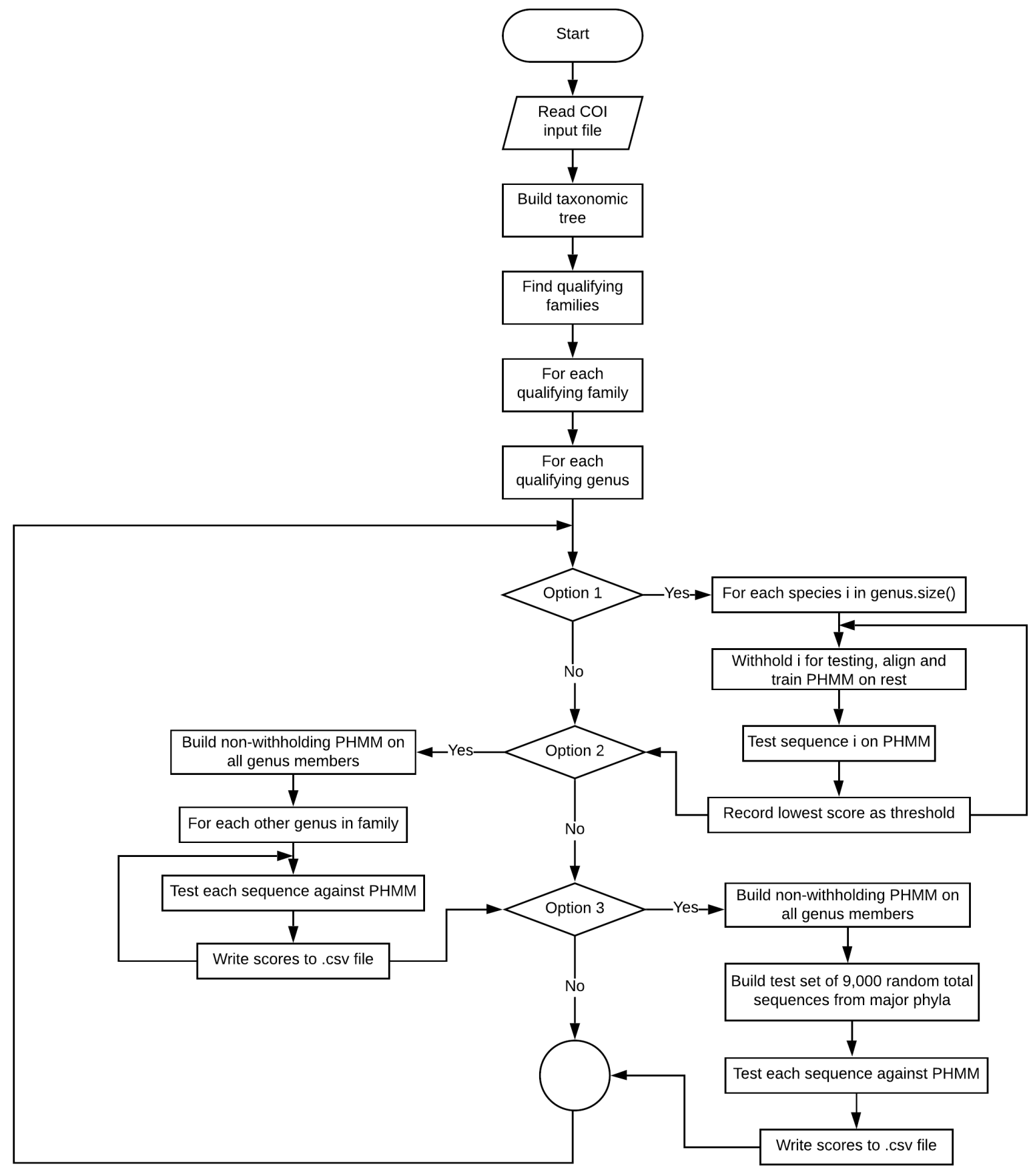

Fig. 3. Project conceptual flowchart. 


\section{Chapter 5: Hardware, Protocol, and Results}

This chapter briefly describes the machines and protocol used for this project, depicts the obtained results through a plot and table for each genus, and summarizes the results with a precision and recall table.

\section{Hardware}

The results of this project were computed on 3 machines:

1. Sager personal laptop (Windows 10)

2. ACER Predator (Ubuntu)

3. The San José State University (SJSU) College of Science High Performance Computing (HPC) system

\section{Protocol}

Because different instances of this project were executed on 3 separate machines, and because the SJSU HPC system does not provide MySQL, all results were written to .csv files and manually combined and analyzed. These .csv files are available upon request.

\section{Results}

Table IV lists general information about each genus that are useful when considering the performance of their profile hidden Markov model (PHMM). It is important to note that the 
columns "minimum length" and "maximum length" do not represent the length requirements, nor the minimum and maximum length that are possible over all organisms in the specified genus. Instead, these columns simply represent the shortest and longest sequence lengths that were represented in our COI dataset. Similarly, the column "average length" contains the calculation of the average length out of the sequences in our input set. These values are subject to change with future additions to our COI database.

All results following are listed in alphabetical order by family then genus. The sizes of the genus, family member genera, and non-member test set are included in the x-axis labels of each chart.

It is necessary to note that the threshold for the genus was not always determined by its lowest score. This is because there were occurrences of single, outlier scores that were not representative of the majority of the genus. For example, in the family Agabini and the genus Agabus, the minimum score (and therefore threshold) would have been -526.6488 . The next lowest score was -448.7219 . However, these scores were only applicable to 2 sequences; all other 24 sequences scored between approximately -77 and -144 . Because these "lowest scores" were not representative of the much larger majority, the third lowest score, -144.18347 , was chosen as the threshold. The result tables in the next subsections will include notes of the lowest scores that were, for this reason, not chosen as thresholds. Adjusted thresholds are marked with an asterisk when listed in the tables. All thresholds were manually selected. 
Accuracy for genus members against their own genus PHMM (after being withheld from training) was calculated by considering the number of members that scored higher than the thresholds selected for their genus. If the threshold was not adjusted, the accuracy was $100 \%$ because the minimum score was chosen as the lowest possible score that a genus member received. 
TABLE IV

GENERAL GENUS INFORMATION

\begin{tabular}{|c|c|c|c|c|c|c|}
\hline Family & Genus & $\begin{array}{l}\text { Minimum } \\
\text { Length }\end{array}$ & $\begin{array}{l}\text { Maximum } \\
\text { Length }\end{array}$ & Average Length & $\begin{array}{l}\text { Standard } \\
\text { deviation }\end{array}$ & Size \\
\hline Acanthosomatidae & Acanthosoma & 609 & 618 & 614.8333 & 4.34450 & 12 \\
\hline Acanthosomatidae & Elasmucha & 600 & 654 & 618.2308 & 12.2144 & 13 \\
\hline Agabini & Agabus & 555 & 826 & 764.6154 & 65.15893 & 26 \\
\hline Agabini & Ilybius & 695 & 826 & 771.0526 & 54.9196 & 19 \\
\hline Agabini & Platynectes & 661 & 826 & 728.0526 & 41.7392 & 19 \\
\hline Agelenidae & Malthonica & 471 & 471 & 471 & 0 & 11 \\
\hline Agelenidae & Sinocoelotes & 1037 & 1194 & 1183.2667 & 40.4660 & 15 \\
\hline Agelenidae & Tegenaria & 325 & 1194 & 706.7407 & 326.8221 & 27 \\
\hline Harpalini & Harpalus & 400 & 1255 & 780.7857 & 150.5935 & 28 \\
\hline Harpalini & Ophonus & 819 & 819 & 819 & 0 & 11 \\
\hline Hydraenidae & Hydraena & 368 & 826 & 738.2159 & 125.0870 & 88 \\
\hline Hydraenidae & Limnebius & 411 & 826 & 750.2308 & 119.6566 & 52 \\
\hline Hydraenidae & Ochthebius & 615 & 829 & 805.1266 & 56.1971 & 79 \\
\hline Hydropsychinae & Ceratopsyche & 351 & 737 & 601.25 & 105.5287 & 20 \\
\hline Hydropsychinae & Cheumatopsyche & 323 & 658 & 627.6945 & 78.8064 & 36 \\
\hline Hydropsychinae & Hydromanicus & 517 & 658 & 641.9231 & 39.7397 & 13 \\
\hline Hydropsychinae & Hydropsyche & 320 & 764 & 624.9492 & 69.1160 & 59 \\
\hline Hydropsychinae & Orthopsyche & 566 & 658 & 652.25 & 23 & 16 \\
\hline Hydropsychinae & Potamyia & 408 & 658 & 633.7692 & 70.1738 & 13 \\
\hline Ophiolepididae & Ophiolepis & 632 & 1431 & 1105.4166 & 402.413 & 12 \\
\hline Ophiolepididae & Ophiomusium & 656 & 1431 & 1374.1333 & 198.7331 & 15 \\
\hline Triozidae & Myotrioza & 426 & 427 & 426.9333 & 0.25820 & 15 \\
\hline Triozidae & Pariaconus & 467 & 472 & 471.6923 & 1.0107 & 26 \\
\hline Triozidae & Trioza & 400 & 649 & 472.1429 & 46.7678 & 21 \\
\hline Vespinae & Vespa & 403 & 1424 & 906.3637 & 334.3257 & 11 \\
\hline Vespinae & Vespula & 408 & 1096 & 602.3846 & 230.05997 & 13 \\
\hline
\end{tabular}




\section{Acanthosomatidae}

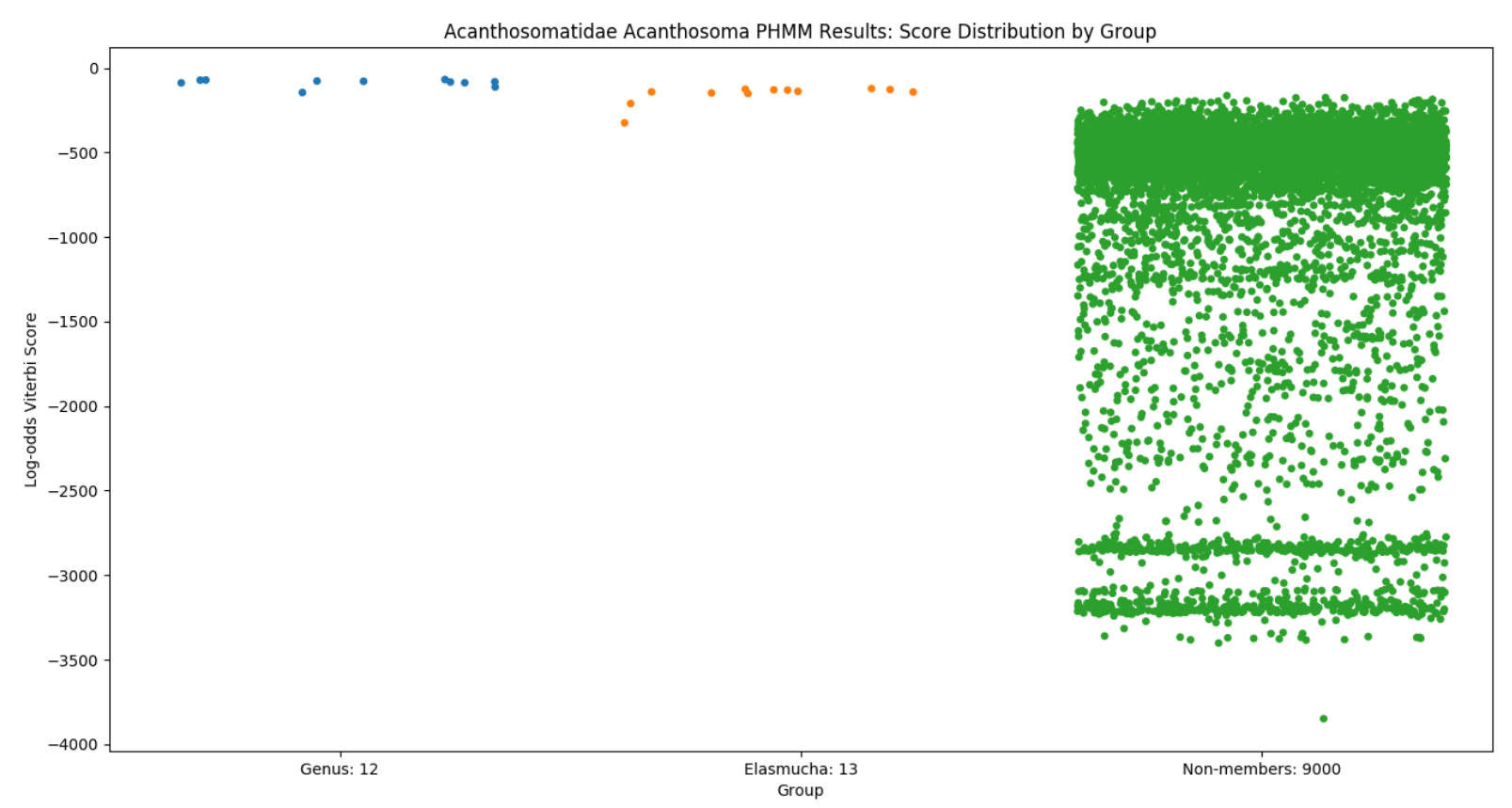

Fig. 4. Results from a profile hidden Markov model trained on Acanthosomatidae Acanthosoma.

TABLE V

ACANTHOSOMATIDAE ACANTHOSOMA RESULTS SUMMARY

\begin{tabular}{|c|c|c|c|c|c|}
\hline \multirow[b]{2}{*}{ Testing } & \multicolumn{5}{|c|}{ Acanthosomatidae Acanthosoma (PHMM) } \\
\hline & $\begin{array}{c}\text { Minimum score } \\
\text { (threshold for self) }\end{array}$ & Maximum score & Size & \# Classified correctly & Accuracy \\
\hline Acanthosoma (self) & -145.2787 & -68.6533 & 12 & 12 & $100 \%$ \\
\hline Elasmucha & -324.8599 & -117.3755 & 13 & 5 & $38.46 \%$ \\
\hline Non-members & -3849.2414 & -145.5663 & 9000 & 9000 & $100 \%$ \\
\hline
\end{tabular}




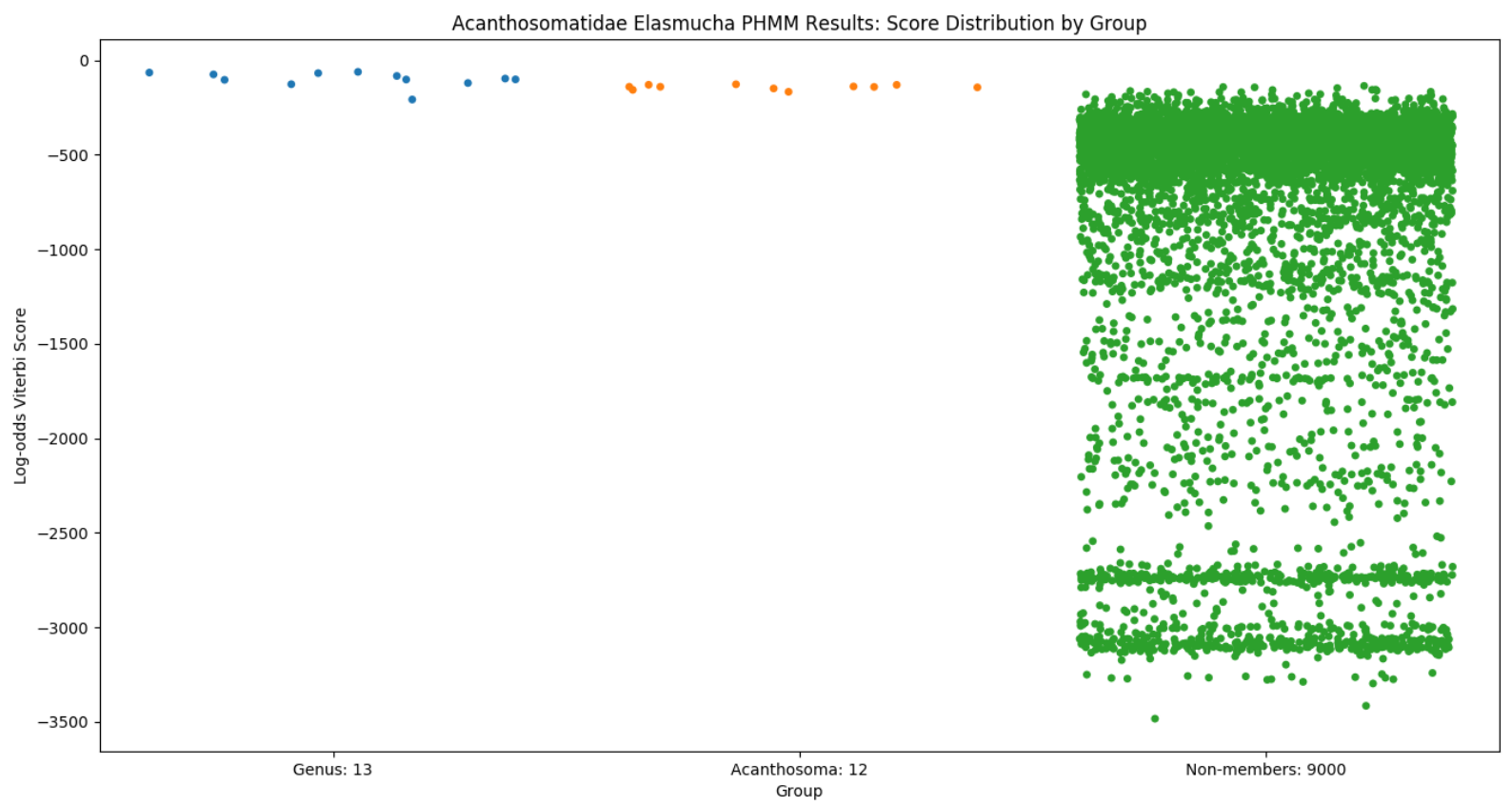

Fig. 5. Results from a profile hidden Markov model trained on Acanthosomatidae Elasmucha.

TABLE VI

ACANTHOSOMATIDAE ELASMUCHA RESULTS SUMMARY

\begin{tabular}{|c|c|c|c|c|c|}
\hline \multirow[b]{2}{*}{ Testing } & \multicolumn{5}{|c|}{ Acanthosomatidae Elasmucha (РНMМ) } \\
\hline & $\begin{array}{l}\text { Minimum score } \\
\text { (threshold for self) }\end{array}$ & Maximum score & Size & \# Classified correctly & Accuracy \\
\hline Elasmucha (self) & -207.9205 & -54.6273 & 13 & 13 & $100 \%$ \\
\hline Acanthosoma & -324.8599 & -117.3755 & 12 & 5 & $35.71 \%$ \\
\hline Non-members & -3483.7305 & -133.7704 & 9000 & 8953 & $99.48 \%$ \\
\hline
\end{tabular}




\section{Agabini}

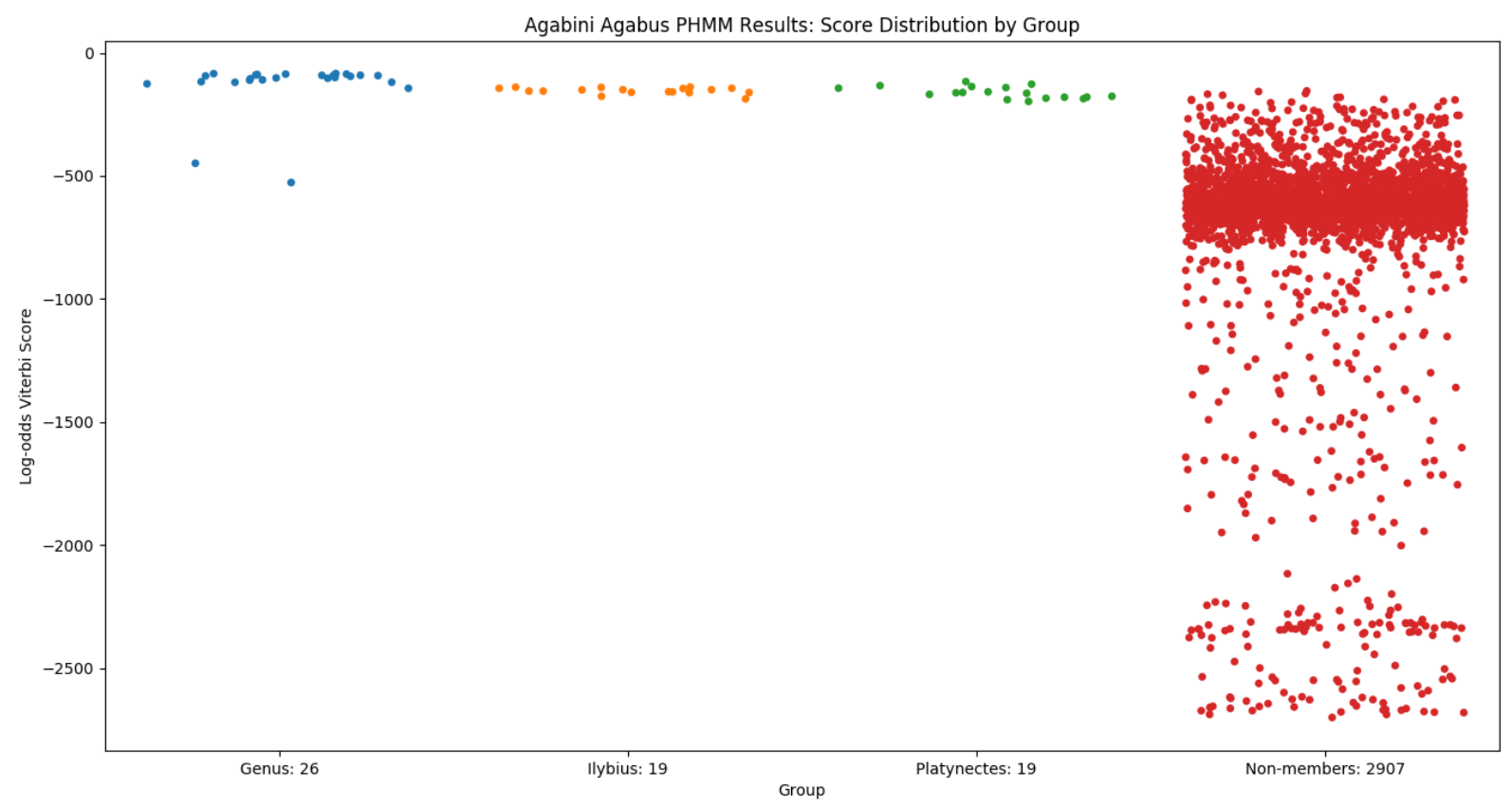

Fig. 6. Results from a profile hidden Markov model trained on Agabini Agabus.

TABLE VII

AGABINI AGABUS RESULTS SUMMARY

\begin{tabular}{|c|c|c|c|c|c|}
\hline \multirow{2}{*}{ Testing } & \multicolumn{5}{|c|}{ Agabini Agabus (PHMM) } \\
\cline { 2 - 6 } & $\begin{array}{c}\text { Minimum score } \\
\text { (threshold for self) }\end{array}$ & Maximum score & Size & \# Classified correctly & Accuracy \\
\hline Agabus (self) & $-144.1835^{*}$ & -77.4202 & 26 & 24 & $92.31 \%$ \\
\hline Ilybius & -186.9433 & -133.1017 & 19 & 13 & $68.42 \%$ \\
\hline Platynectes & -197.6052 & -114.3496 & 19 & 12 & $63.16 \%$ \\
\hline Non-members & -2699.1710 & -151.4505 & 2907 & 2907 & $100 \%$ \\
\hline
\end{tabular}

*Disqualified scores: $-526.6488,-448.7219$. 
Agabini llybius PHMM Results: Score Distribution by Group

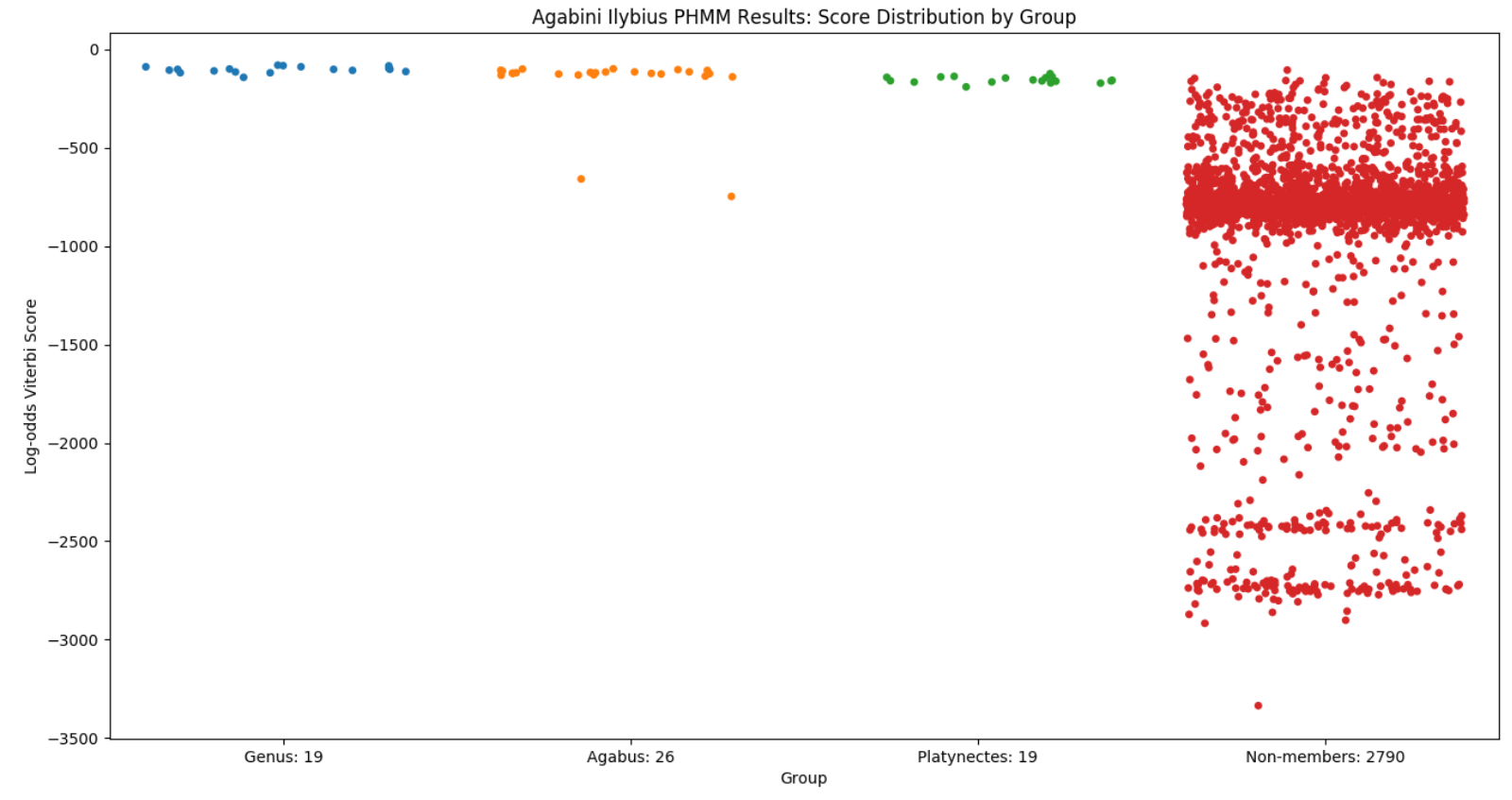

Fig. 7. Results from a profile hidden Markov model trained on Agabini Ilybius.

TABLE VIII

AGABINI ILYBIUS RESULTS SUMMARY

\begin{tabular}{|c|c|c|c|c|}
\hline \multirow{2}{*}{ Testing } & \multicolumn{2}{|c|}{ Agabini Ilybius (PHMM) } \\
\cline { 2 - 5 } & Minimum score & Maximum score & Size & \# Classified correctly \\
& (threshold for self) & -78.42338 & 19 & \\
\hline Ilybius (self) & -141.9061 & -97.9478 & 26 & \\
\hline Agabus & -747.0695 & -120.0789 & 19 & 2 \\
\hline Platynectes & -190.2305 & -104.8589 & $27.69 \%$ \\
\hline
\end{tabular}


Agabini Platynectes PHMM Results: Score Distribution by Group

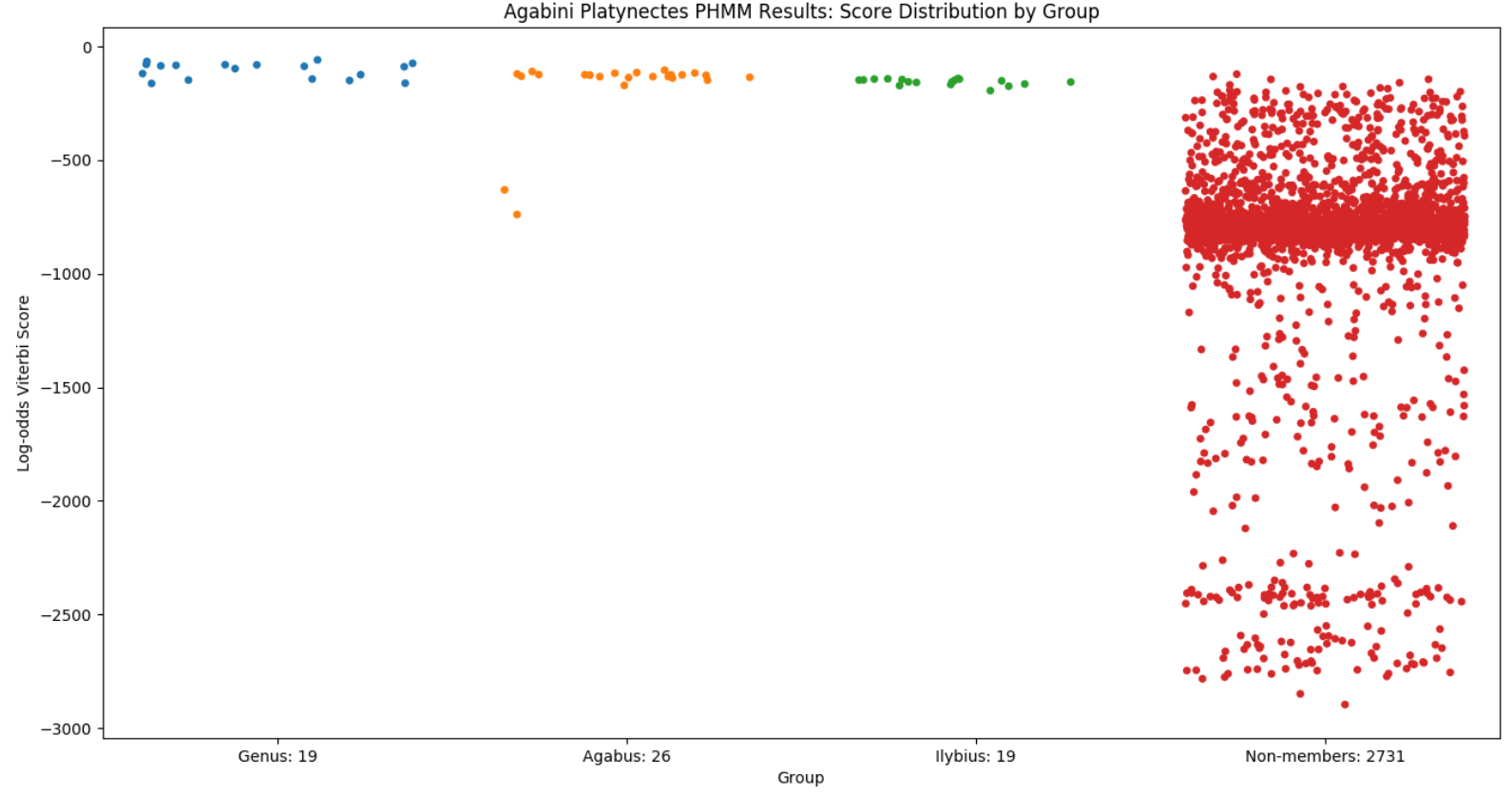

Fig. 8. Results from a profile hidden Markov model trained on Agabini Platynectes.

TABLE IX

AGABINI PLATYNECTES RESULTS SUMMARY

\begin{tabular}{|c|c|c|c|c|c|}
\hline \multirow[b]{2}{*}{ Testing } & \multicolumn{5}{|c|}{ Agabini Platynectes (PHMM) } \\
\hline & $\begin{array}{l}\text { Minimum score } \\
\text { (threshold for self) }\end{array}$ & Maximum score & Size & \# Classified correctly & Accuracy \\
\hline Platynectes (self) & -162.2267 & -57.5830 & 19 & 19 & $100 \%$ \\
\hline Agabus & -739.3214 & -100.7318 & 26 & 4 & $15.38 \%$ \\
\hline Ilybius & -194.04908 & -139.2075 & 19 & 5 & $26.32 \%$ \\
\hline Non-members & -2896.4042 & -81.80703 & 2731 & 2725 & $99.78 \%$ \\
\hline
\end{tabular}




\section{Agelenidae}

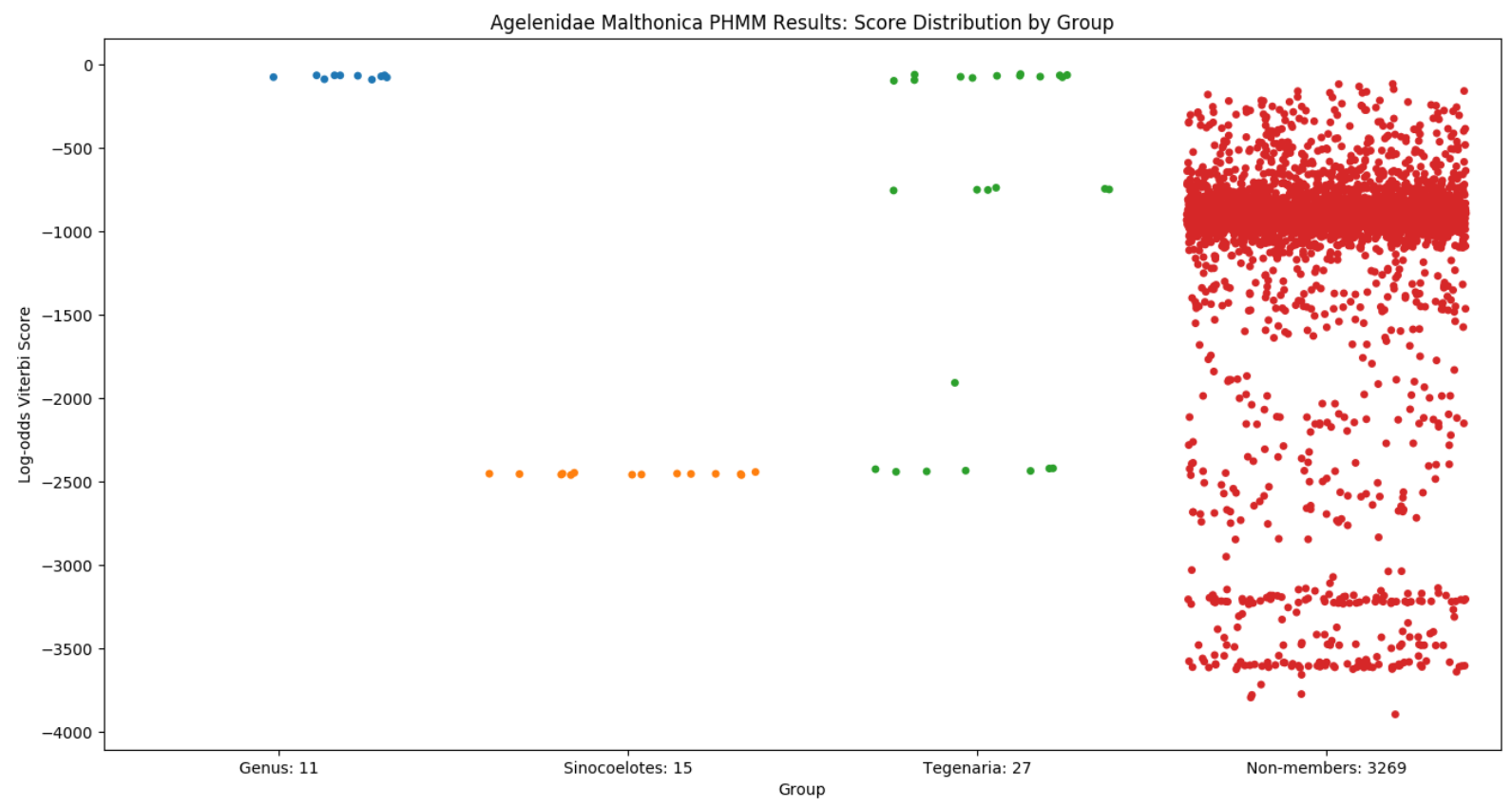

Fig. 9. Results from a profile hidden Markov model trained on Agelenidae Malthonica.

TABLE X

AGELENIDAE MALTHONICA RESULTS SUMMARY

\begin{tabular}{|c|c|c|c|c|c|}
\hline \multirow{2}{*}{ Testing } & \multicolumn{5}{|c|}{ Agelenidae Malthonica (PHMM) } \\
\cline { 2 - 6 } & $\begin{array}{c}\text { Minimum score } \\
\text { (threshold for self) }\end{array}$ & Maximum score & Size & \# Classified correctly & Accuracy \\
\hline Malthonica (self) & -88.8033 & -58.0055 & 11 & 11 & $100 \%$ \\
\hline Sinocoelotes & -2459.0996 & -1952.8030 & 15 & 15 & $100 \%$ \\
\hline Tegenaria & -2439.4216 & -54.0495 & 27 & 16 & $59.26 \%$ \\
\hline Non-members & -3893.3856 & -105.5835 & 3269 & 3269 & $100 \%$ \\
\hline
\end{tabular}


Agelenidae Sinocoelotes PHMM Results: Score Distribution by Group

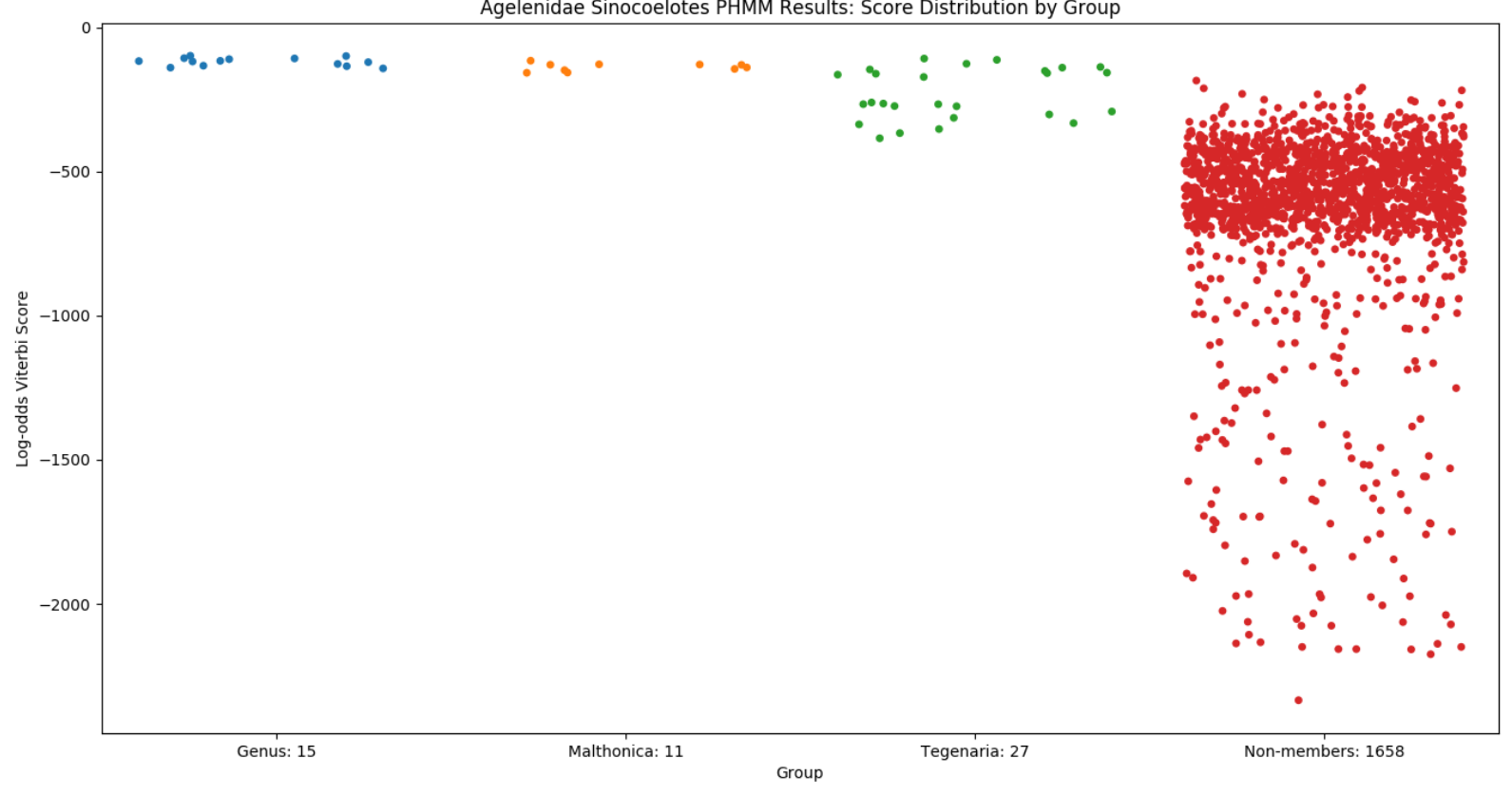

Fig. 10. Results from a profile hidden Markov model trained on Agelenidae Sinocoelotes.

TABLE XI

AGELENIDAE SINOCOELOTES RESULTS SUMMARY

\begin{tabular}{|c|c|c|c|c|c|}
\hline \multirow{2}{*}{ Testing } & \multicolumn{4}{|c|}{ Agelenidae Sinocoelotes (PHMM) } \\
\cline { 2 - 6 } & $\begin{array}{c}\text { Minimum score } \\
\text { (threshold for self) }\end{array}$ & Maximum score & Size & \# Classified correctly & Accuracy \\
\hline Sinocoelotes (self) & -142.3542 & -95.0836 & 15 & 15 & $100 \%$ \\
\hline Malthonica & -157.5088 & -100.9536 & 11 & 4 & $36.37 \%$ \\
\hline Tegenaria & -384.8089 & -96.6936 & 27 & 21 & $77.78 \%$ \\
\hline Non-members & -2334.3002 & -148.1235 & 1658 & 1658 & $100 \%$ \\
\hline
\end{tabular}


Agelenidae Tegenaria PHMM Results: Score Distribution by Group

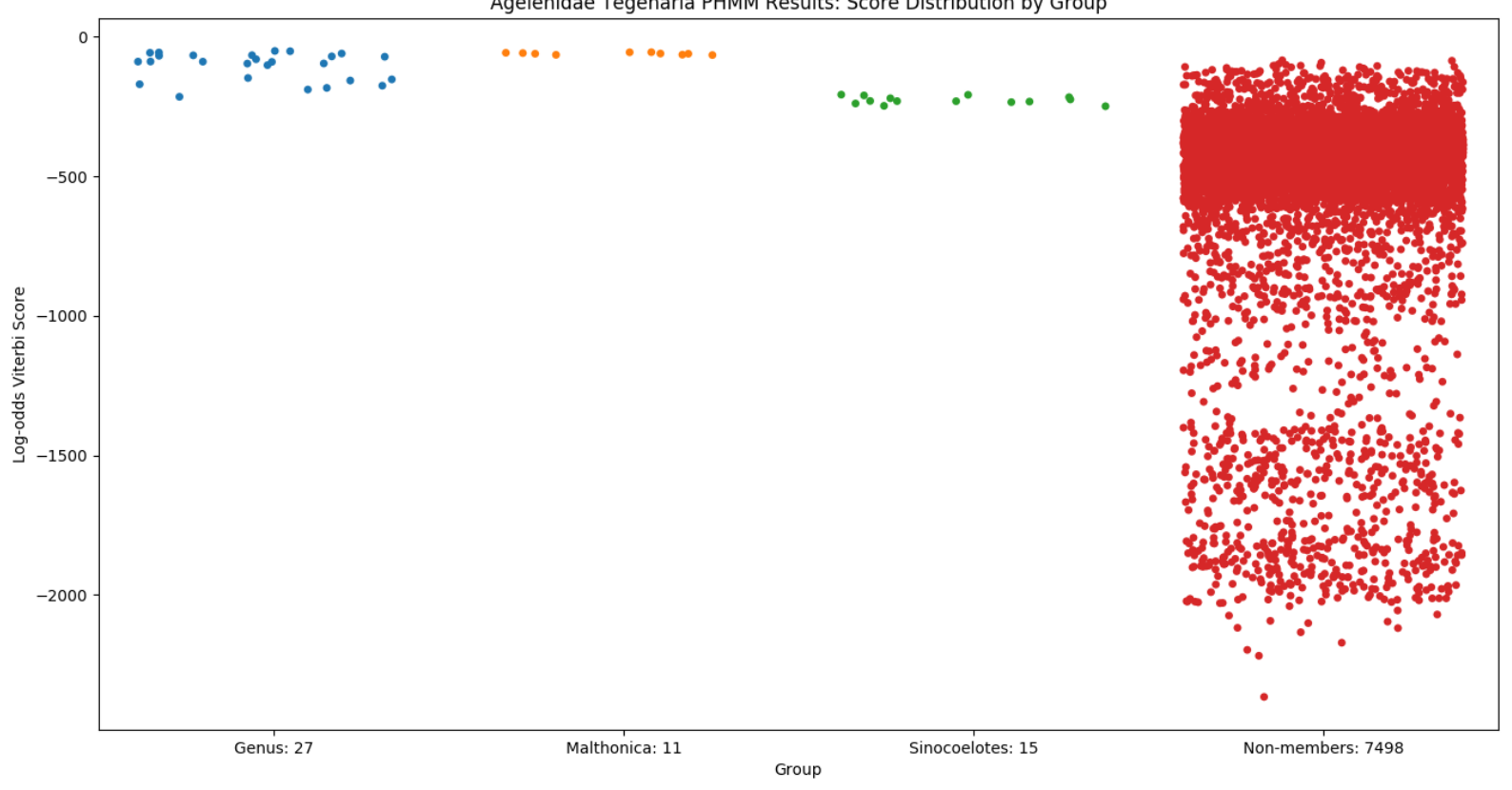

Fig 11. Results from a profile hidden Markov model trained on Agelenidae Tegenaria.

TABLE XII

AGELENIDAE TEGENARIA RESULTS SUMMARY

\begin{tabular}{|c|c|c|c|c|c|}
\hline \multirow{2}{*}{ Testing } & \multicolumn{5}{|c|}{ Agelenidae Tegenaria (PHMM) } \\
\cline { 2 - 6 } & $\begin{array}{c}\text { Minimum score } \\
\text { (threshold for self) }\end{array}$ & Maximum score & Size & \# Classified correctly & Accuracy \\
\hline Tegenaria (self) & -215.1850 & -48.3479 & 27 & 27 & $100 \%$ \\
\hline Malthonica & -65.6623 & -49.6836 & 11 & 0 & $0 \%$ \\
\hline Sinocoelotes & -249.0387 & -204.6202 & 15 & 11 & $73.33 \%$ \\
\hline Non-members & -2366.2047 & -84.3493 & 7498 & 7262 & $96.85 \%$ \\
\hline
\end{tabular}




\section{Harpalini}

Harpalini Harpalus PHMM Results: Score Distribution by Group

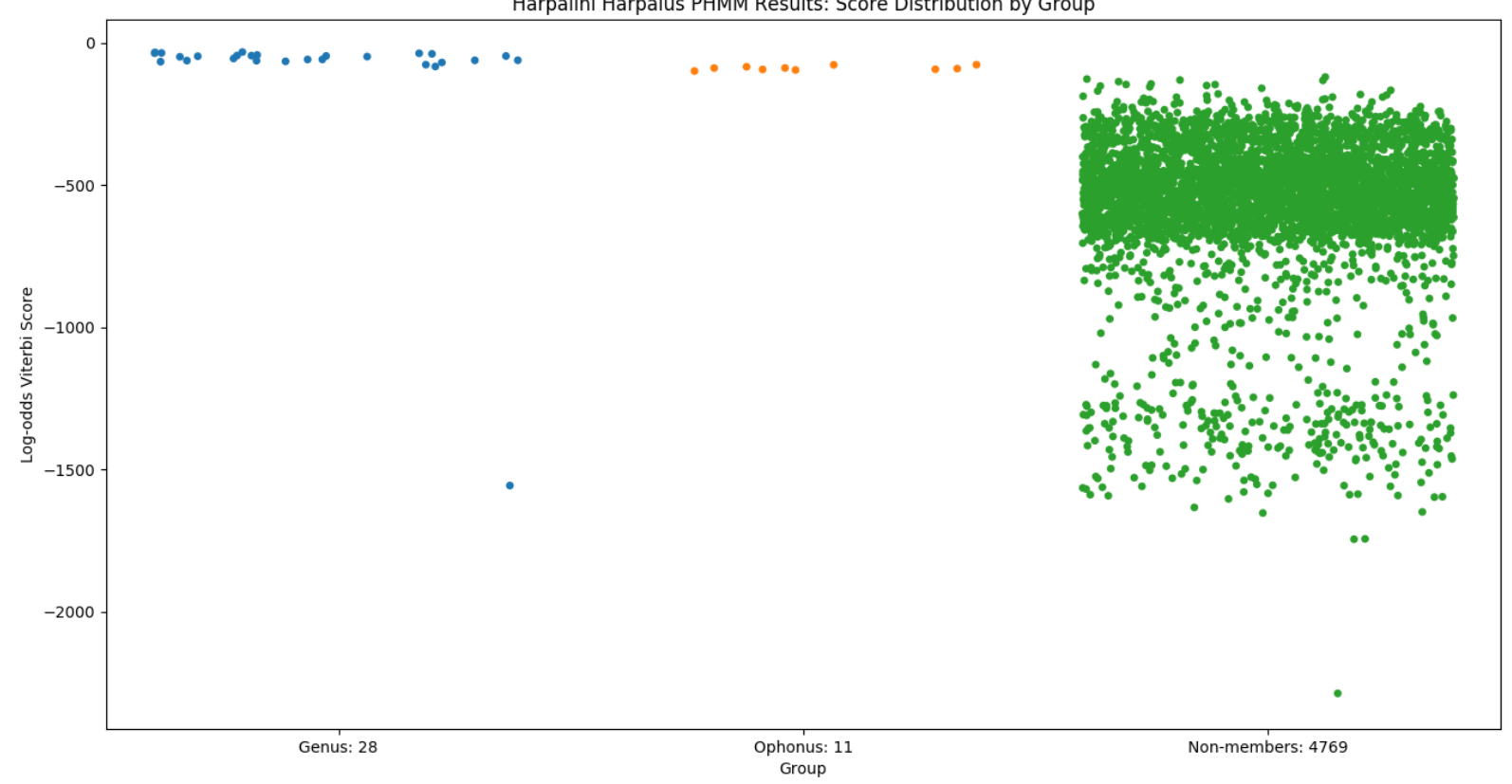

Fig. 12. Results from a profile hidden Markov model trained on Harpalini Harpalus.

TABLE XIII

HARPALINI HARPALUS RESULTS SUMMARY

\begin{tabular}{|c|c|c|c|c|c|}
\hline \multirow{2}{*}{ Testing } & \multicolumn{4}{|c|}{ Harpalini Harpalus (PHMM) } \\
\cline { 2 - 6 } & $\begin{array}{c}\text { Minimum score } \\
\text { (threshold for self) }\end{array}$ & $\begin{array}{c}\text { Maximum } \\
\text { score }\end{array}$ & Size & \multicolumn{2}{|c|}{ \# Classified } \\
correctly & Accuracy \\
\hline Harpalus (self) & $-83.6572^{*}$ & -55.4468 & 28 & 27 & $96.43 \%$ \\
\hline Ophonus & -99.6018 & -75.1085 & 11 & 8 & $72.72 \%$ \\
\hline Non-members & -2287.9793 & -119.1523 & 4769 & 4769 & $100 \%$ \\
\hline
\end{tabular}

*Disqualified score: -1557.1130 . 
Harpalini Ophonus PHMM Results: Score Distribution by Group

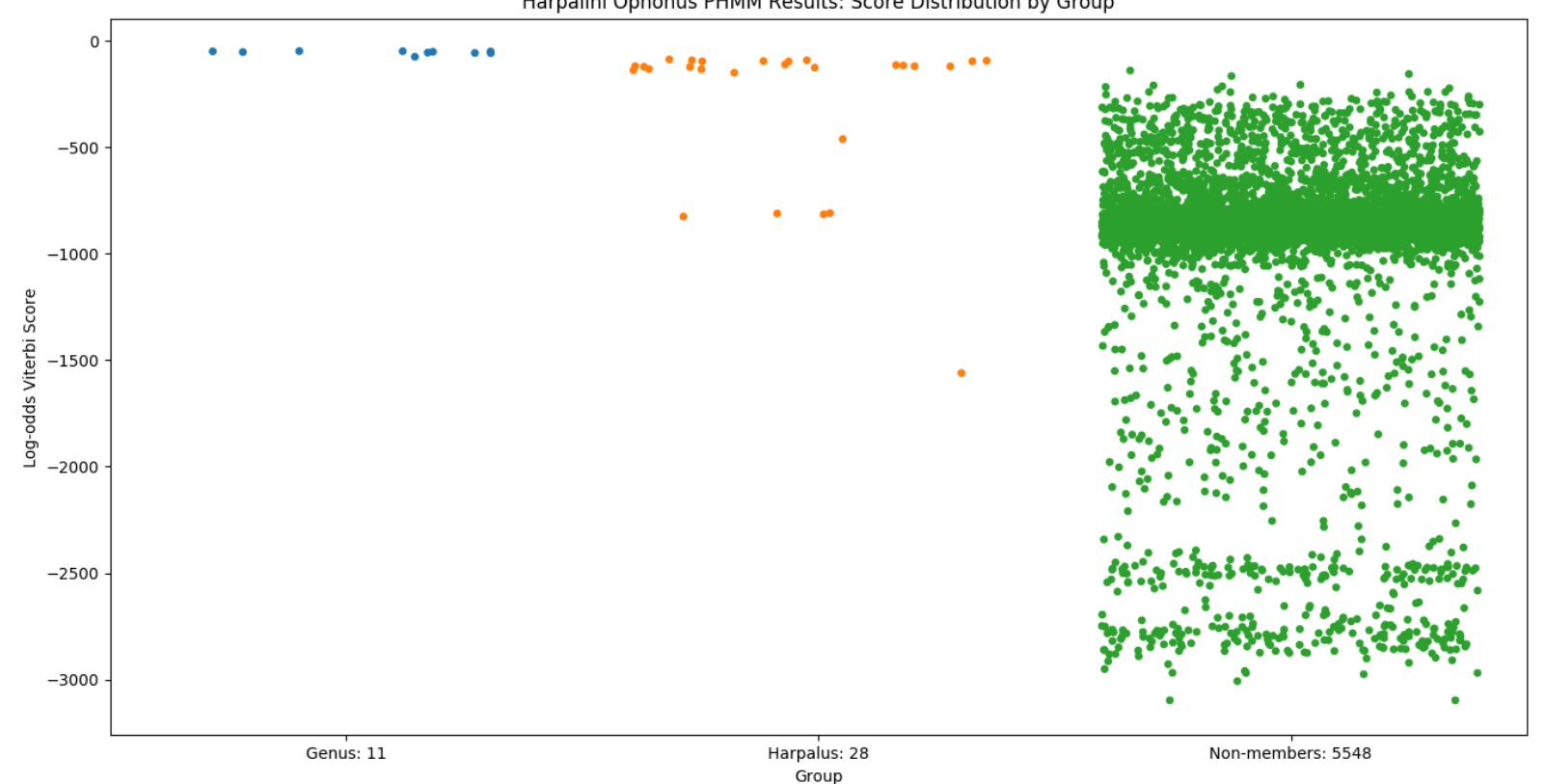

Fig. 13. Results from a profile hidden Markov model trained on Harpalini Ophonus.

TABLE XIV

HARPALINI OPHONUS RESULTS SUMMARY

\begin{tabular}{|c|c|c|c|c|c|}
\hline \multirow[b]{2}{*}{ Testing } & \multicolumn{5}{|c|}{ Harpalini Ophonus (PHMM) } \\
\hline & $\begin{array}{l}\text { Minimum score } \\
\text { (threshold for self) }\end{array}$ & $\begin{array}{l}\text { Maximum } \\
\text { score }\end{array}$ & Size & \# Classified correctly & Accuracy \\
\hline Ophonus (self) & -75.3018 & -42.9332 & 11 & 11 & $100 \%$ \\
\hline Harpalus & -1560.7978 & -57.6815 & 28 & 27 & $96.43 \%$ \\
\hline Non-members & -3097.5086 & -124.6532 & 5548 & 5548 & $100 \%$ \\
\hline
\end{tabular}




\section{Hydraenidae}

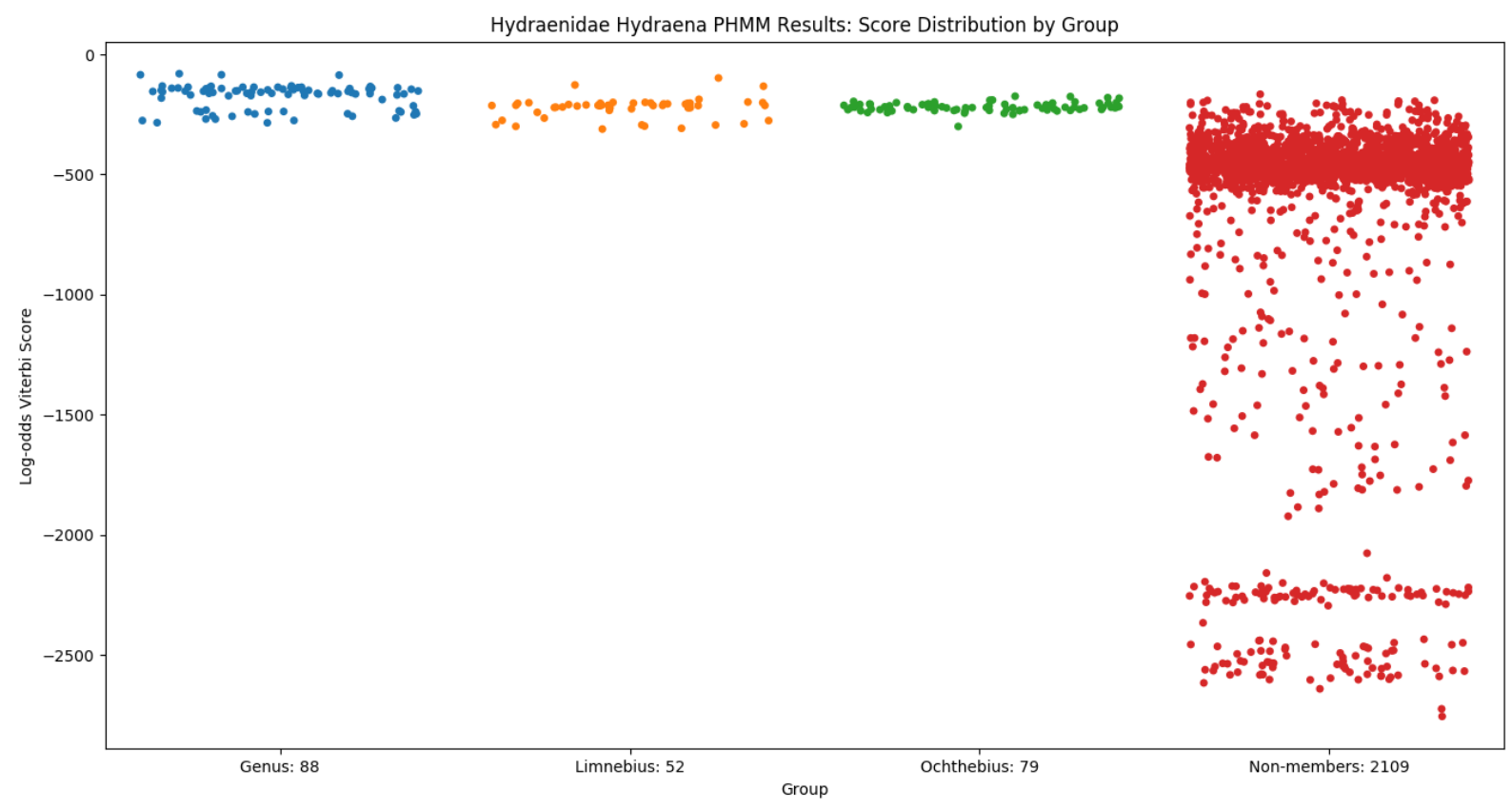

Fig. 14. Results from a profile hidden Markov model trained on Hydraenidae Hydraena.

TABLE XV

HYDRAENIDAE HYDRAENA RESULTS SUMMARY

\begin{tabular}{|c|c|c|c|c|c|}
\hline \multirow{2}{*}{ Testing } & \multicolumn{4}{|c|}{ Hydraenidae Hydraena (PHMM) } \\
\cline { 2 - 6 } & $\begin{array}{c}\text { Minimum score } \\
\text { (threshold for self) }\end{array}$ & Maximum score & Size & \# Classified correctly & Accuracy \\
\hline Hydraena (self) & -284.7792 & -71.8597 & 88 & 88 & $100 \%$ \\
\hline Limnebius & -310.8380 & -78.1422 & 52 & 8 & $15.38 \%$ \\
\hline Ochthebius & -299.9345 & -165.3602 & 79 & 1 & $1.27 \%$ \\
\hline Non-members & -2755.1463 & -155.7135 & 2109 & 2030 & $96.25 \%$ \\
\hline
\end{tabular}




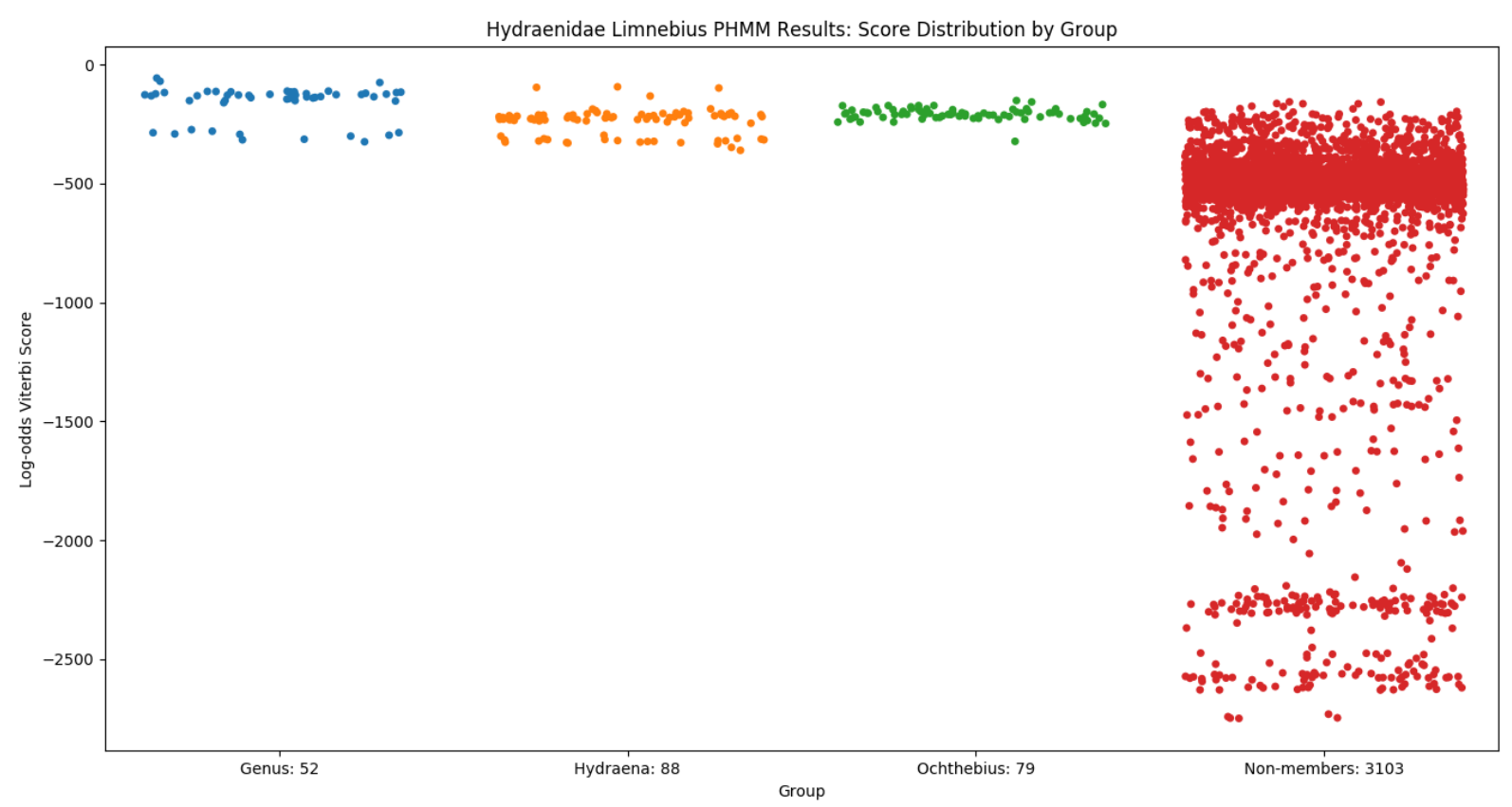

Fig. 15. Results from a profile hidden Markov model trained on Hydraenidae Limnebius.

TABLE XVI

HYDRAENIDAE LIMNEBIUS RESULTS SUMMARY

\begin{tabular}{|c|c|c|c|c|c|}
\hline \multirow[b]{2}{*}{ Testing } & \multicolumn{5}{|c|}{ Hydraenidae Limnebius (PHMM) } \\
\hline & $\begin{array}{c}\text { Minimum score } \\
\text { (threshold for self) }\end{array}$ & Maximum score & Size & \# Classified correctly & Accuracy \\
\hline Limnebius (self) & $-160.0001^{*}$ & -54.9159 & 52 & 41 & $78.85 \%$ \\
\hline Hydraena & -360.1851 & -84.4259 & 88 & 83 & $94.32 \%$ \\
\hline Ochthebius & -322.4946 & -135.7819 & 79 & 79 & $100 \%$ \\
\hline Non-members & -2749.5237 & -154.7686 & 3103 & 3100 & $99.9 \%$ \\
\hline
\end{tabular}

*Disqualified scores: -273.2946, -279.7449, -285.7762, -286.1403, -291.1698, -293.0570,

$$
-296.6379,-300.4486,-313.6522,-316.0419,-324.1376 .
$$


Hydraenidae Ochthebius PHMM Results: Score Distribution by Group

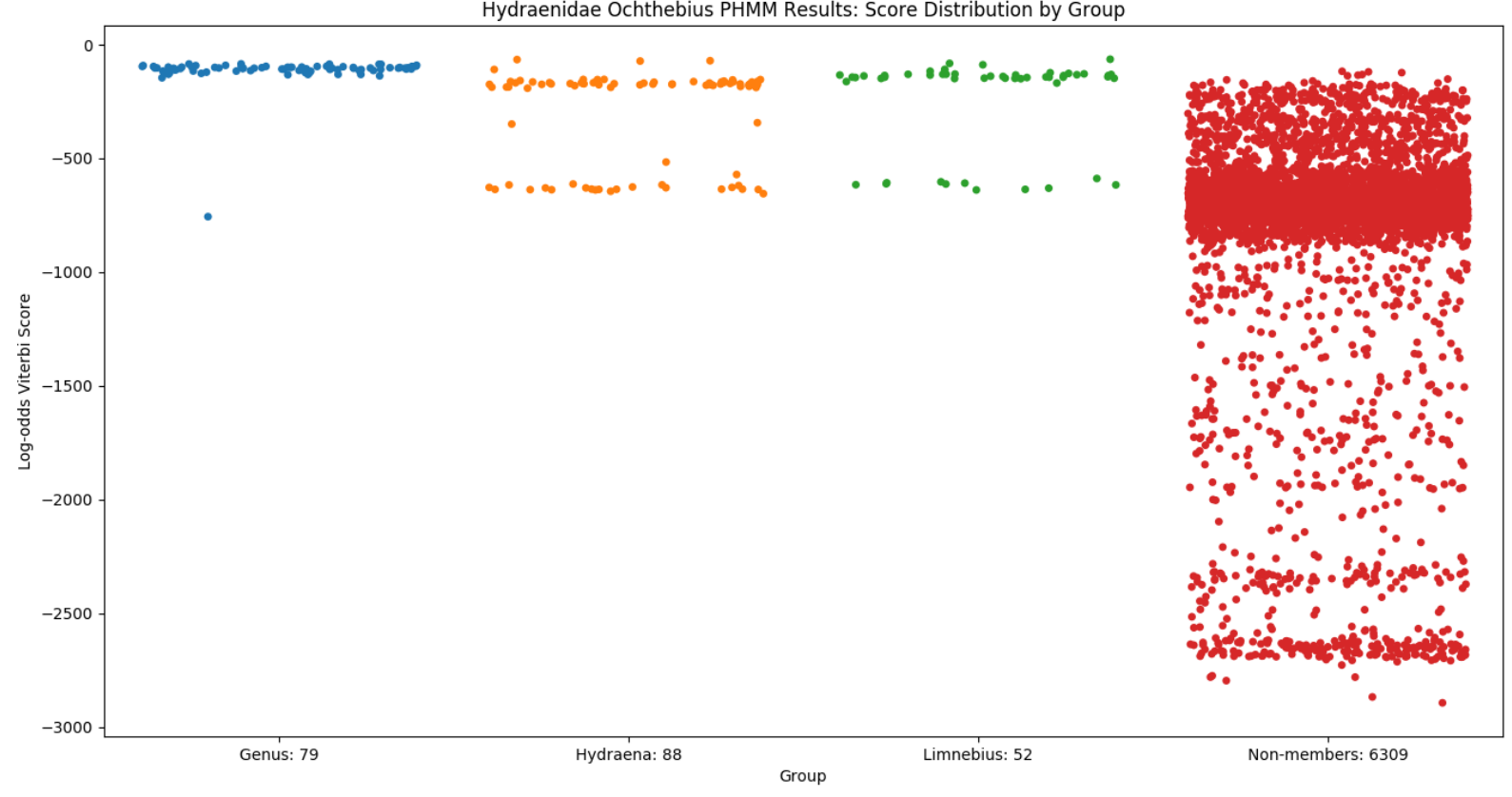

Fig. 16. Results from a profile hidden Markov model trained on Hydraenidae Ochthebius.

TABLE XVII

HYDRAENIDAE OCHTHEBIUS RESULTS SUMMARY

\begin{tabular}{|c|c|c|c|c|c|}
\hline \multirow{2}{*}{ Testing } & \multicolumn{5}{|c|}{ Hydraenidae Ochthebius (PHMM) } \\
\cline { 2 - 6 } & $\begin{array}{c}\text { Minimum score } \\
\text { (threshold for self) }\end{array}$ & Maximum score & Size & \# Classified correctly & Accuracy \\
\hline Ochthebius (self) & $-145.7303^{*}$ & -75.2817 & 79 & 78 & $98.73 \%$ \\
\hline Hydraena & -655.0737 & -65.5207 & 88 & 93 & $34.32 \%$ \\
\hline Limnebius & -638.6286 & -53.9566 & 52 & 20 & $38.46 \%$ \\
\hline Non-members & -2892.6540 & -115.6407 & 6309 & 6302 & $99.89 \%$ \\
\hline
\end{tabular}

*Disqualified score: -756.0902. 
TOWARD ON-DEMAND PROFILE HIDDEN MARKOV MODELS FOR GENETIC BARCODE IDENTIFICATION

\section{Hydropsychinae}

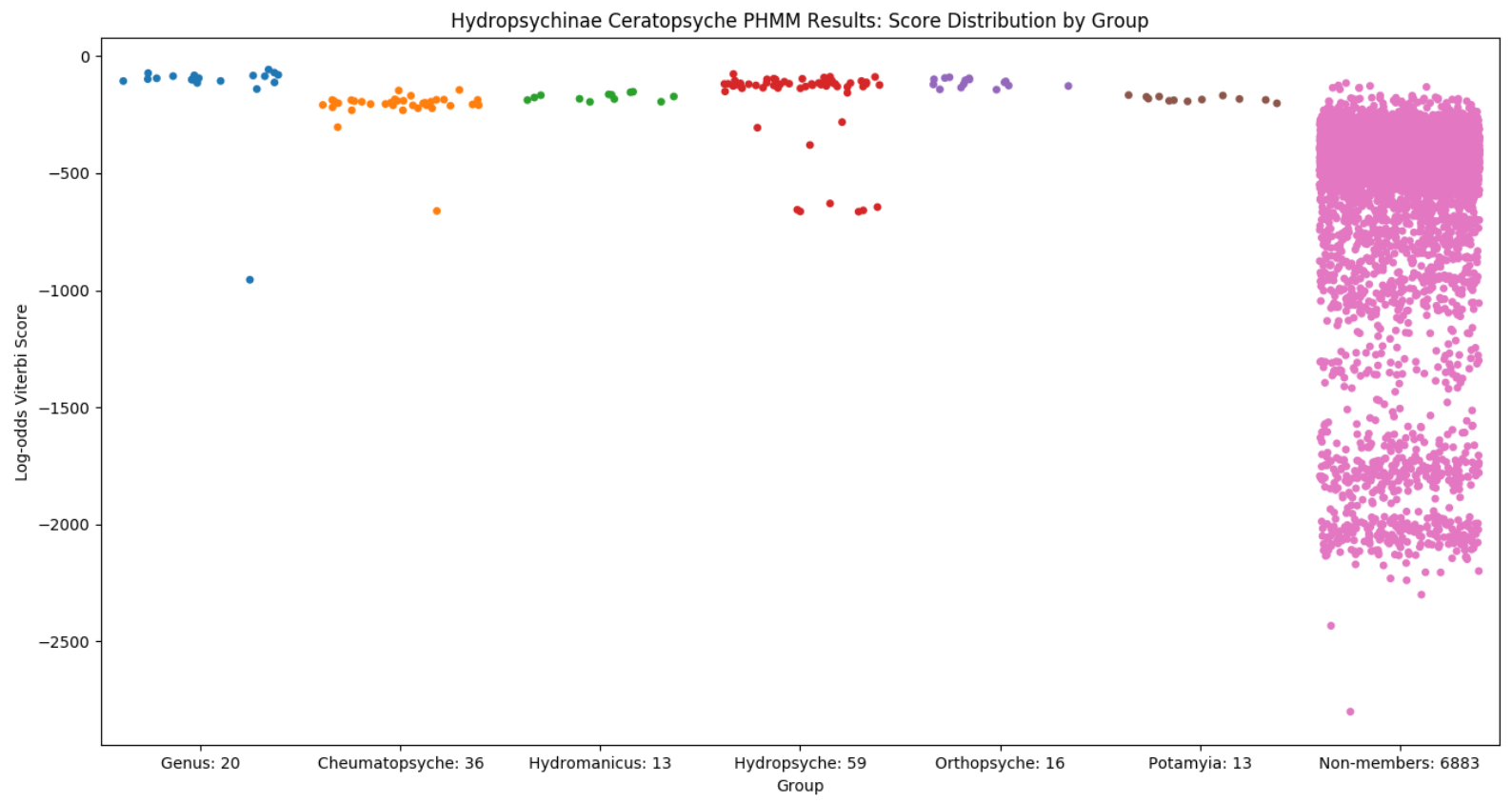

Fig. 17. Results from a profile hidden Markov model trained on Hydropsychinae Ceratopsyche. 
TABLE XVIII

HYDROPSYCHINAE CERATOPSYCHE RESULTS SUMMARY

\begin{tabular}{|c|c|c|c|c|c|}
\hline \multirow{2}{*}{ Testing } & \multicolumn{5}{|c|}{ Hydropsychinae Ceratopsyche (PHMM) } \\
\cline { 2 - 6 } & $\begin{array}{c}\text { Minimum score } \\
\text { (threshold for self) }\end{array}$ & $\begin{array}{c}\text { Maximum } \\
\text { score }\end{array}$ & Size & \# Classified correctly & Accuracy \\
\hline Ceratopsyche (self) & $-140.4110^{*}$ & -54.8585 & 20 & 19 & $95 \%$ \\
\hline Cheumatopsyche & -661.3194 & -110.1625 & 36 & 35 & $97.22 \%$ \\
\hline Hydromanicus & -195.2657 & -133.6520 & 13 & 12 & $93.21 \%$ \\
\hline Hydropsyche & -664.4730 & -63.0735 & 59 & 11 & $18.64 \%$ \\
\hline Orthopsyche & -143.0898 & -87.0942 & 16 & 2 & $12.5 \%$ \\
\hline Potamyia & -201.4294 & -115.7971 & 13 & 12 & $93.21 \%$ \\
\hline Non-members & -2800.4680 & -114.1592 & 6883 & 6875 & $99.89 \%$ \\
\hline
\end{tabular}

*Disqualified score: -955.2970. 
Hydropsychinae Cheumatopsyche PHMM Results: Score Distribution by Group

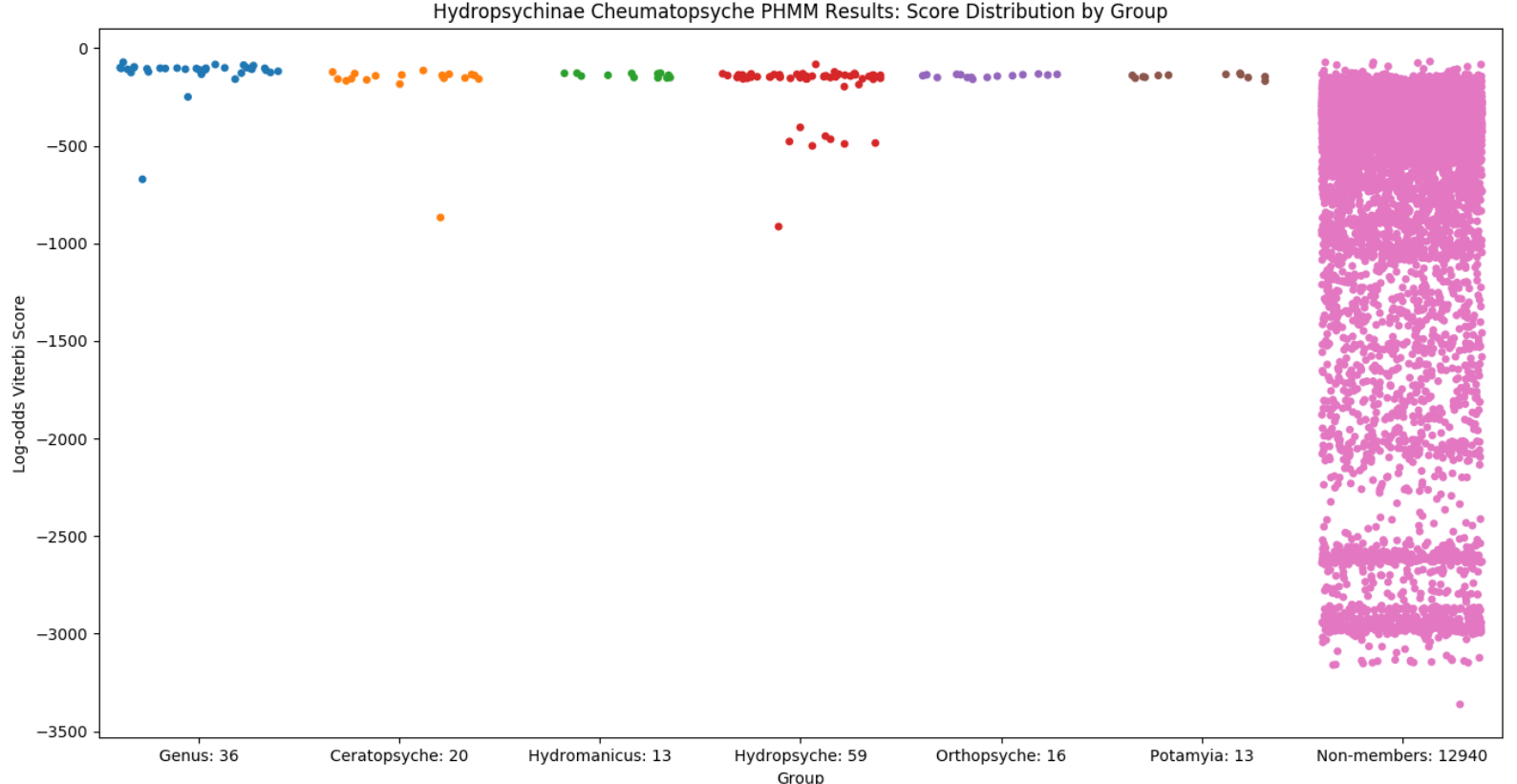

Fig. 18. Results from a profile hidden Markov model trained on Hydropsychinae Cheumatopsyche.

TABLE XIX

HYDROPSYCHINAE CHEUMATOPSYCHE RESULTS SUMMARY

\begin{tabular}{|c|c|c|c|c|c|}
\hline \multirow[b]{2}{*}{ Testing } & \multicolumn{5}{|c|}{ Hydropsychinae Cheumatopsyche (PHMM) } \\
\hline & $\begin{array}{c}\text { Minimum score } \\
\text { (threshold for self) }\end{array}$ & $\begin{array}{c}\text { Maximum } \\
\text { score }\end{array}$ & Size & \# Classified correctly & Accuracy \\
\hline Cheumatopsyche (self) & $-134.0977^{*}$ & -53.5881 & 36 & 33 & $91.67 \%$ \\
\hline Ceratopsyche & -868.1133 & -90.3373 & 20 & 15 & $75 \%$ \\
\hline Hydromanicus & -153.1423 & -120.4040 & 13 & 7 & $53.85 \%$ \\
\hline Hydropsyche & -914.6981 & -63.5376 & 59 & 52 & $88.14 \%$ \\
\hline Orthopsyche & -160.2116 & -124.9142 & 16 & 14 & $87.5 \%$ \\
\hline Potamyia & -169.1443 & -126.3407 & 13 & 11 & $84.62 \%$ \\
\hline Non-members & -3362.5040 & -66.4741 & 12940 & 12915 & $99.81 \%$ \\
\hline
\end{tabular}

*Disqualified scores: -672.2618, -250.2219, -159.1693. 
Hydropsychinae Hydromanicus PHMM Results: Score Distribution by Group

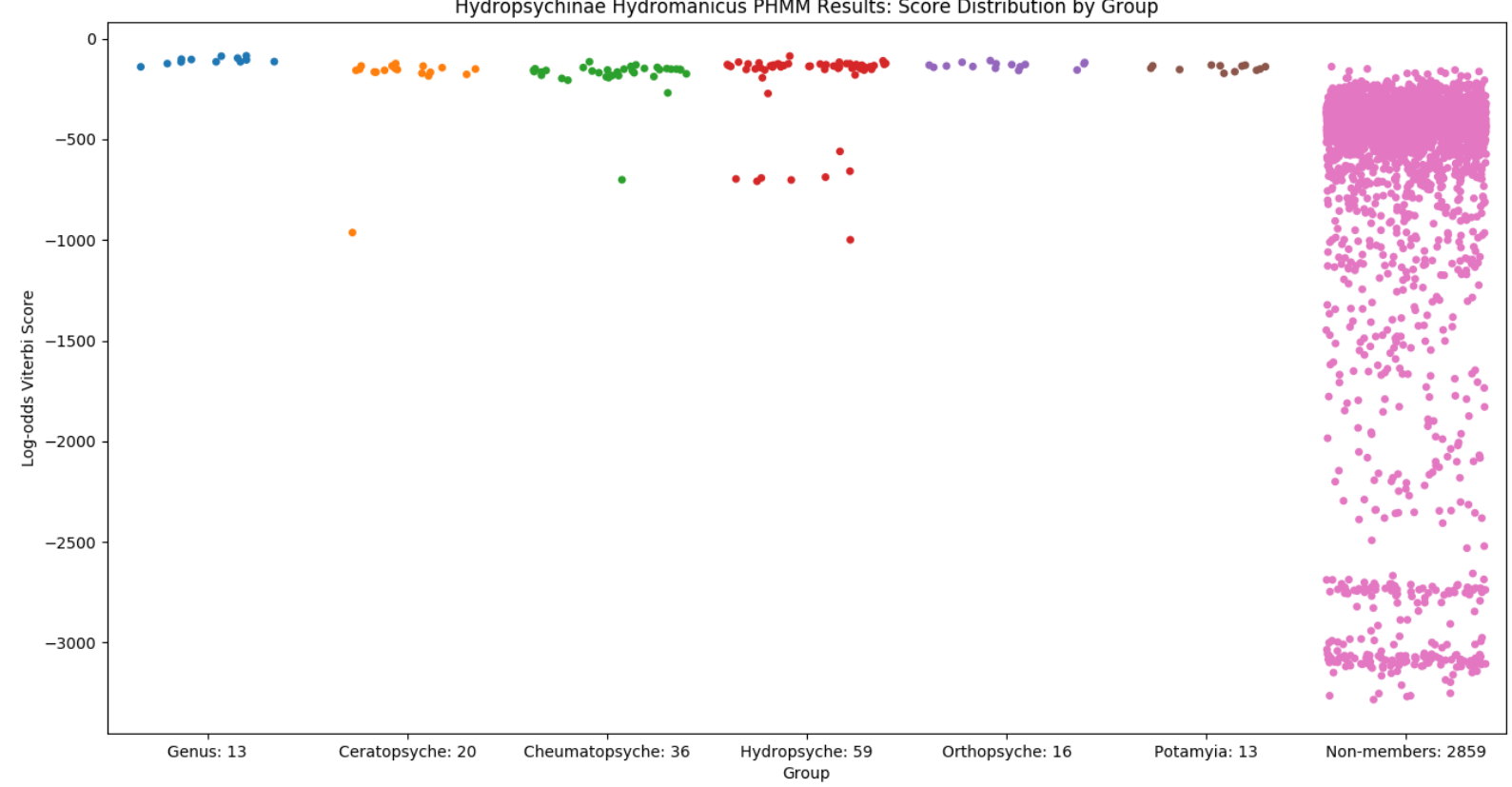

Fig. 19. Results from a profile hidden Markov model trained on Hydropsychinae Hydromanicus.

TABLE XX

HYDROPSYCHINAE HYDROMANICUS RESULTS SUMMARY

\begin{tabular}{|c|c|c|c|c|c|}
\hline \multirow[b]{2}{*}{ Testing } & \multicolumn{5}{|c|}{ Hydropsychinae Hydromanicus (PHMM) } \\
\hline & $\begin{array}{c}\text { Minimum score } \\
\text { (threshold for self) }\end{array}$ & $\begin{array}{l}\text { Maximum } \\
\text { score }\end{array}$ & Size & \# Classified correctly & Accuracy \\
\hline Hydromanicus (self) & -140.2212 & -84.3756 & 13 & 13 & $100 \%$ \\
\hline Ceratopsyche & -963.3288 & -93.3407 & 20 & 15 & $75 \%$ \\
\hline Cheumatopsyche & -701.2450 & -82.9502 & 36 & 32 & $88.9 \%$ \\
\hline Hydropsyche & -999.2702 & -85.5230 & 59 & 27 & $45.77 \%$ \\
\hline Orthopsyche & -158.5835 & -108.7546 & 16 & 4 & $25 \%$ \\
\hline Potamyia & -172.6139 & -130.2208 & 13 & 6 & $46.15 \%$ \\
\hline Non-members & -3283.1875 & -110.3033 & 2859 & 2857 & $99.93 \%$ \\
\hline
\end{tabular}


Hydropsychinae Hydropsyche PHMM Results: Score Distribution by Group

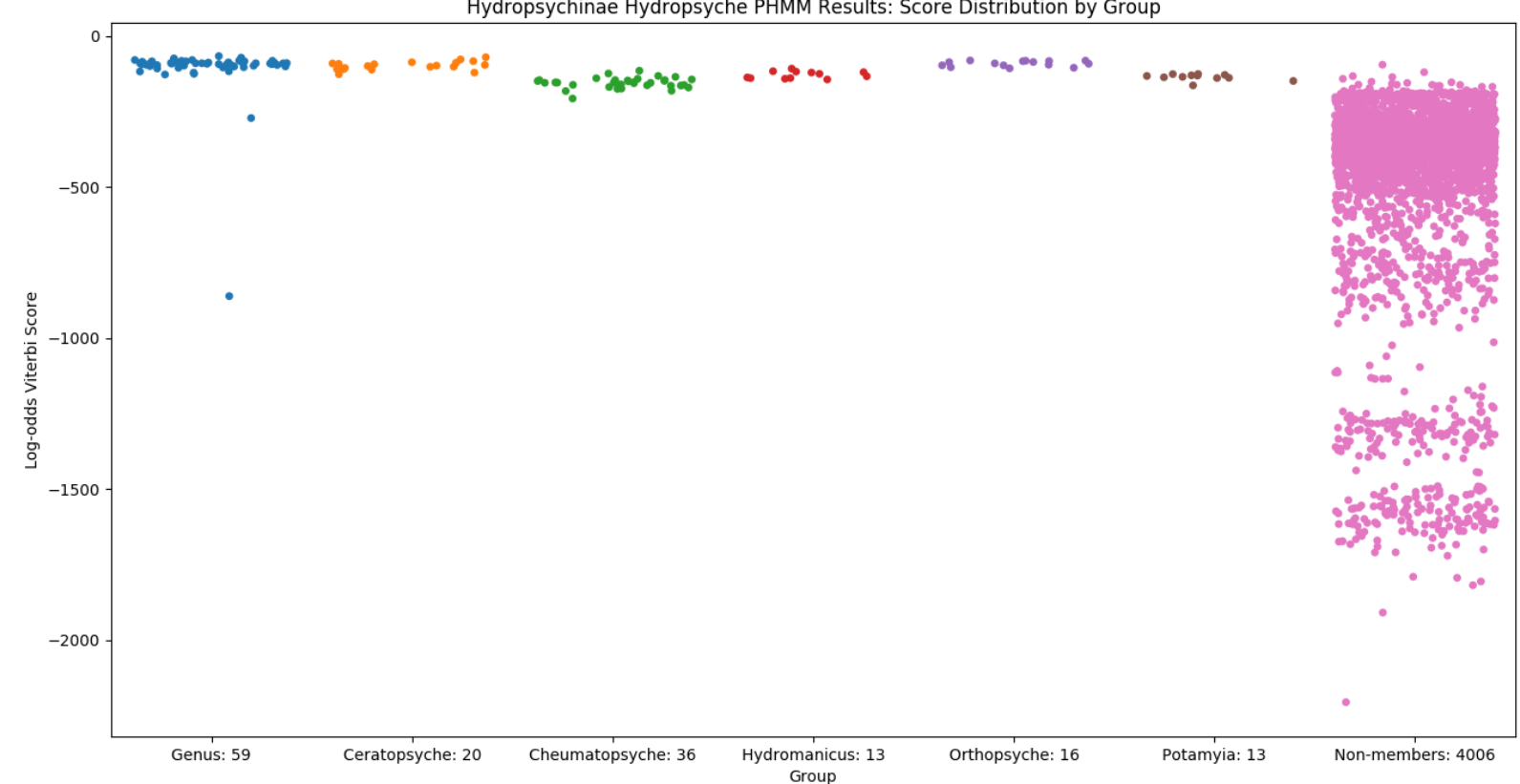

Fig. 20. Results from a profile hidden Markov model trained on Hydropsychinae Hydropsyche.

TABLE XXI

HYDROPSYCHINAE HYDROPSYCHE RESULTS SUMMARY

\begin{tabular}{|c|c|c|c|c|c|}
\hline \multirow[b]{2}{*}{ Testing } & \multicolumn{5}{|c|}{ Hydropsychinae Hydropsyche (PHMM) } \\
\hline & $\begin{array}{l}\text { Minimum score } \\
\text { (threshold for self) }\end{array}$ & $\begin{array}{l}\text { Maximum } \\
\text { score }\end{array}$ & Size & \# Classified correctly & Accuracy \\
\hline Hydropsyche (self) & $-127.1435^{*}$ & -47.1396 & 59 & 57 & $96.61 \%$ \\
\hline Ceratopsyche & -126.2392 & -56.9999 & 20 & 0 & $0 \%$ \\
\hline Cheumatopsyche & -206.6662 & -72.1274 & 36 & 33 & $91.67 \%$ \\
\hline Hydromanicus & -143.5420 & -95.7846 & 13 & 6 & $46.15 \%$ \\
\hline Orthopsyche & -106.7011 & -79.3038 & 16 & 0 & $0 \%$ \\
\hline Potamyia & -163.2032 & -91.1338 & 13 & 10 & $76.92 \%$ \\
\hline Non-members & -2205.5283 & -78.7750 & 4006 & 4003 & $99.93 \%$ \\
\hline
\end{tabular}

*Disqualified score: -861.03739. 
Hydropsychinae Orthopsyche PHMM Results: Score Distribution by Group

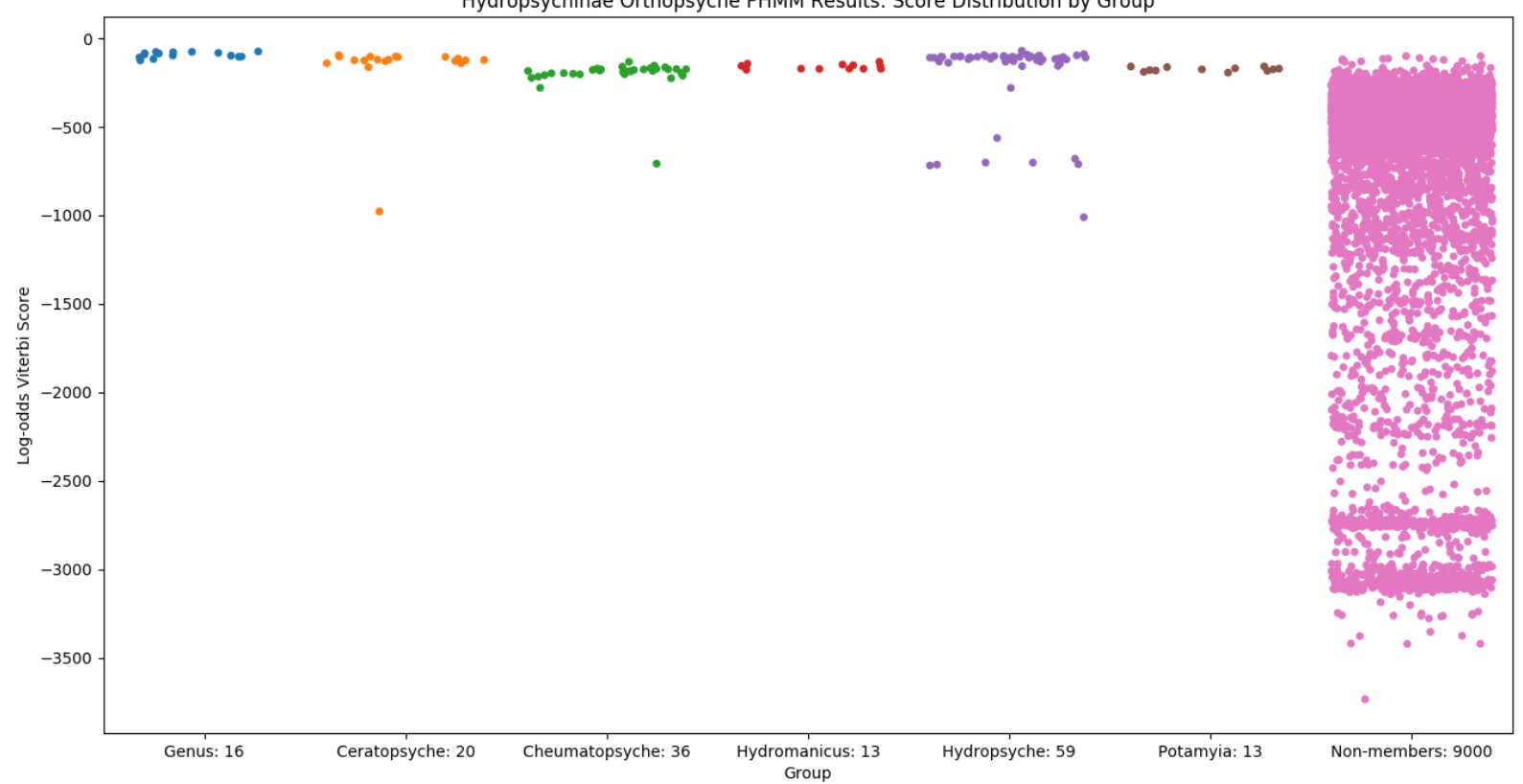

Fig. 21. Results from a profile hidden Markov model trained on Hydropsychinae Orthopsyche.

TABLE XXII

HYDROPSYCHINAE ORTHOPSYCHE RESULTS SUMMARY

\begin{tabular}{|c|c|c|c|c|c|}
\hline \multirow[b]{2}{*}{ Testing } & \multicolumn{5}{|c|}{ Hydropsychinae Orthopsyche (РНММ) } \\
\hline & $\begin{array}{c}\text { Minimum score } \\
\text { (threshold for self) }\end{array}$ & $\begin{array}{l}\text { Maximum } \\
\text { score }\end{array}$ & Size & \# Classified correctly & Accuracy \\
\hline Orthopsyche (self) & -124.062 & -73.5363 & 16 & 16 & $100 \%$ \\
\hline Ceratopsyche & -978.3316 & -58.9259 & 20 & 8 & $40 \%$ \\
\hline Cheumatopsyche & -708.1878 & -97.7470 & 36 & 35 & $97.22 \%$ \\
\hline Hydromanicus & -177.6825 & -115.2416 & 13 & 12 & $92.31 \%$ \\
\hline Hydropsyche & -1010.8450 & -61.9528 & 59 & 17 & $28.81 \%$ \\
\hline Potamyia & -193.3704 & -156.7096 & 13 & 13 & $100 \%$ \\
\hline Non-members & -3734.2816 & -86.8701 & 9000 & 8991 & $99.9 \%$ \\
\hline
\end{tabular}


Hydropsychinae Potamyia PHMM Results: Score Distribution by Group

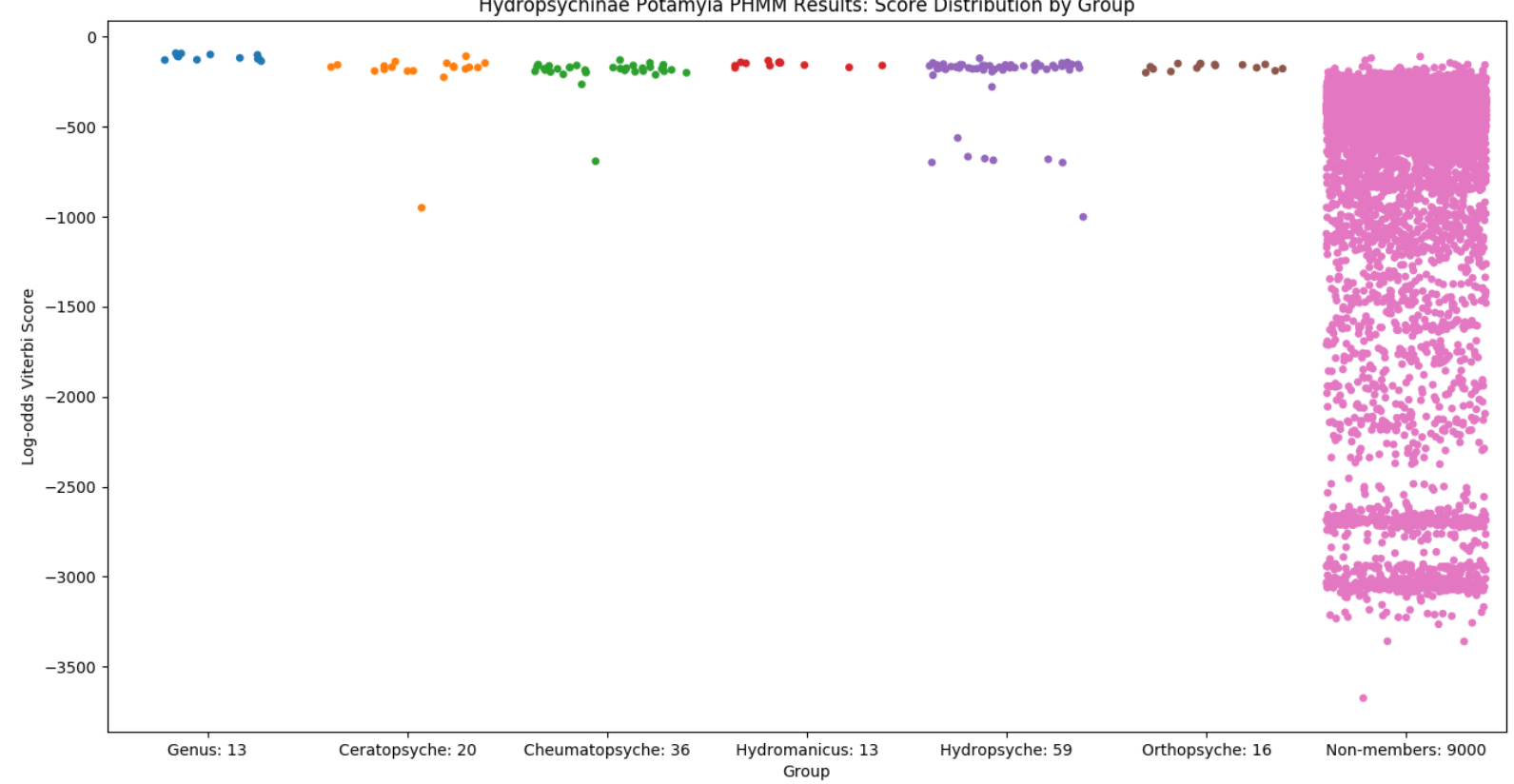

Fig. 22. Results from a profile hidden Markov model trained on Hydropsychinae Potamyia.

TABLE XXIII

HYDROPSYCHINAE POTAMYIA RESULTS SUMMARY

\begin{tabular}{|c|c|c|c|c|c|}
\hline \multirow[b]{2}{*}{ Testing } & \multicolumn{5}{|c|}{ Hydropsychinae Potamyia (PHMM) } \\
\hline & $\begin{array}{c}\text { Minimum score } \\
\text { (threshold for self) }\end{array}$ & $\begin{array}{l}\text { Maximum } \\
\text { score }\end{array}$ & Size & \# Classified correctly & Accuracy \\
\hline Potamyia (self) & -134.8930 & -82.2002 & 13 & 13 & $100 \%$ \\
\hline Ceratopsyche & -950.1965 & -104.2121 & 20 & 18 & $90 \%$ \\
\hline Cheumatopsyche & -691.1670 & -101.0566 & 36 & 34 & $94.44 \%$ \\
\hline Hydromanicus & -172.0796 & -115.2660 & 13 & 11 & $84.62 \%$ \\
\hline Hydropsyche & -1000.8592 & -88.3487 & 59 & 57 & $96.61 \%$ \\
\hline Orthopsyche & -199.0660 & -147.0523 & 16 & 16 & $100 \%$ \\
\hline Non-members & -3675.7010 & -106.7570 & 9000 & 8996 & $99.95 \%$ \\
\hline
\end{tabular}




\section{Ophiolepididae}

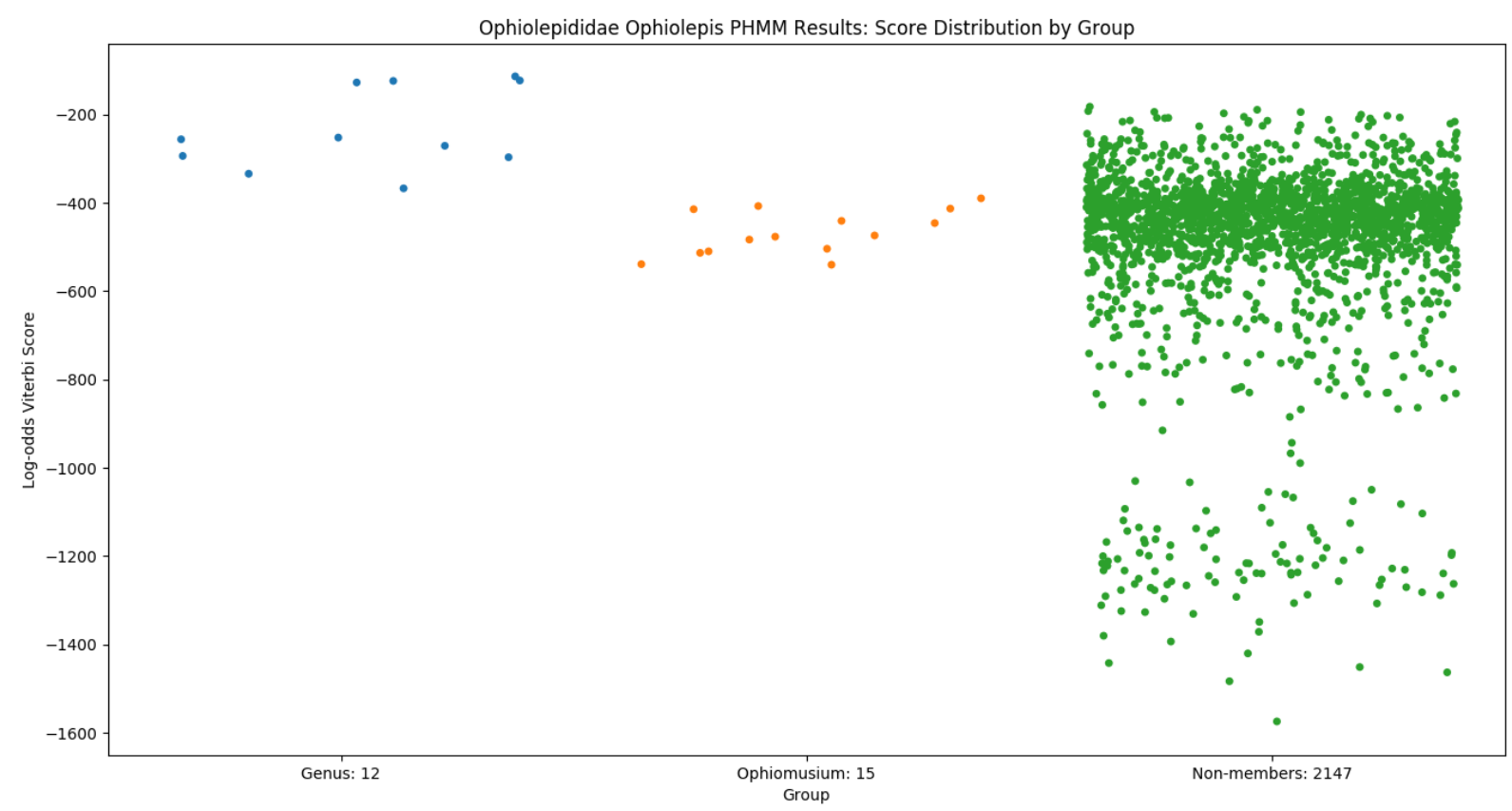

Fig. 23. Results from a profile hidden Markov model trained on Ophiolepididae Ophiolepis.

TABLE XXIV

OPHIOLEPIDIDAE OPHIOLEPIS RESULTS SUMMARY

\begin{tabular}{|c|c|c|c|c|c|}
\hline \multirow{2}{*}{ Testing } & \multicolumn{3}{|c|}{ Ophiolepididae Ophiolepis (PHMM) } \\
\cline { 2 - 6 } & $\begin{array}{c}\text { Minimum score } \\
\text { (threshold for self) }\end{array}$ & $\begin{array}{c}\text { Maximum } \\
\text { score }\end{array}$ & Size & \# Classified correctly & Accuracy \\
\hline Ophiolepis (self) & -367.13160 & -81.4062 & 12 & 12 & $100 \%$ \\
\hline Ophiomusium & -540.0372 & -271.7609 & 15 & 14 & $93.3 \%$ \\
\hline Non-members & -1573.99580 & -172.1904 & 2147 & 1804 & $84.02 \%$ \\
\hline
\end{tabular}




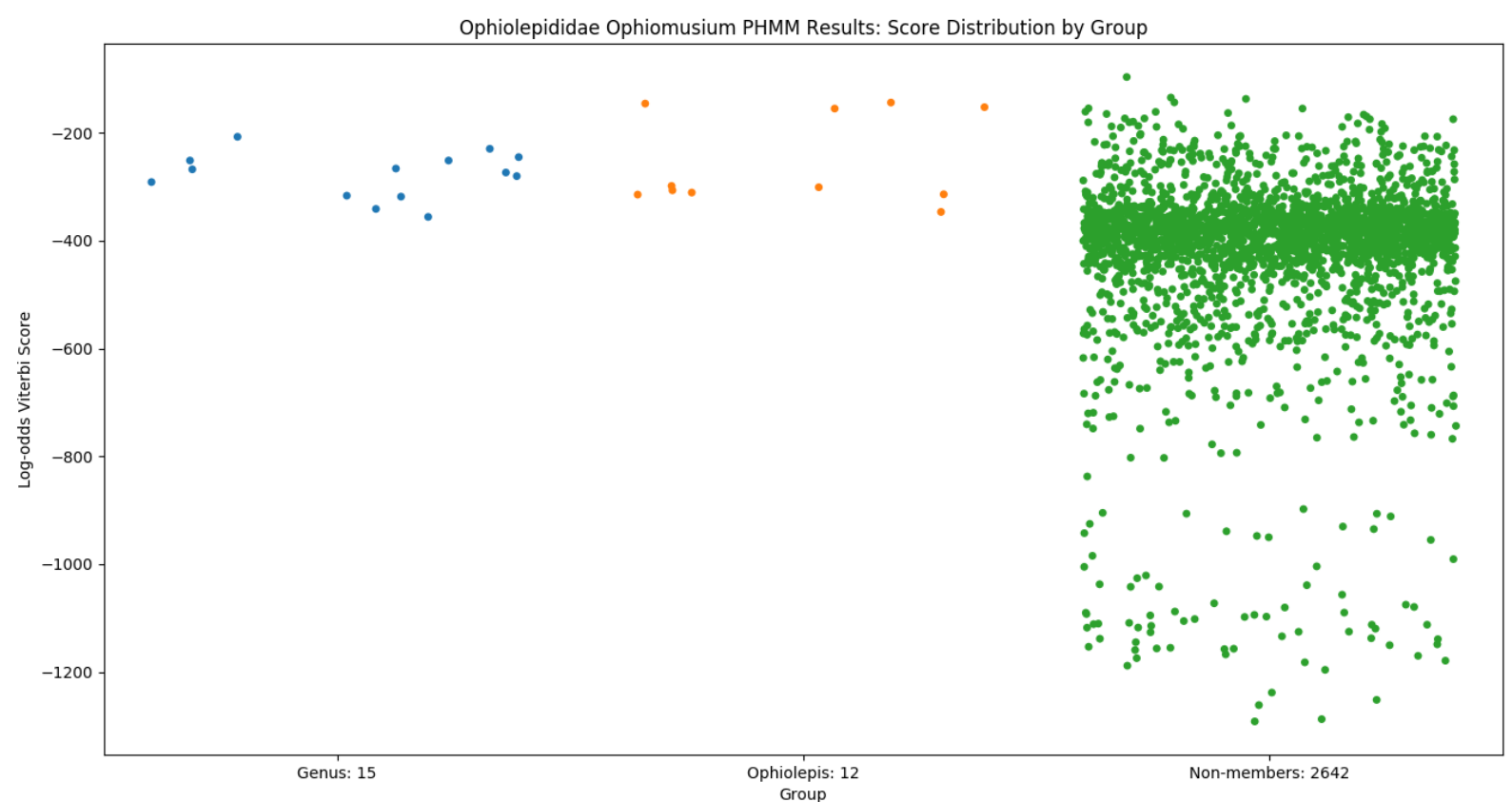

Fig. 24. Results from a profile hidden Markov model trained on Ophiolepididae Ophiomusium.

TABLE XXV

OPHIOLEPIDIDAE OPHIOMUSIUM RESULTS SUMMARY

\begin{tabular}{|c|c|c|c|c|c|}
\hline \multirow[b]{2}{*}{ Testing } & \multicolumn{5}{|c|}{ Ophiolepididae Ophiomusium (РНMM) } \\
\hline & $\begin{array}{l}\text { Minimum score } \\
\text { (threshold for self) }\end{array}$ & $\begin{array}{l}\text { Maximum } \\
\text { score }\end{array}$ & Size & \# Classified correctly & Accuracy \\
\hline Ophiomusium (self) & -355.9895 & -177.9055 & 15 & 15 & $100 \%$ \\
\hline Ophiolepis & -346.7472 & -131.5018 & 12 & 0 & $0 \%$ \\
\hline Non-members & -1291.7723 & -87.2524 & 2642 & 1952 & $73.88 \%$ \\
\hline
\end{tabular}




\section{Triozidae}

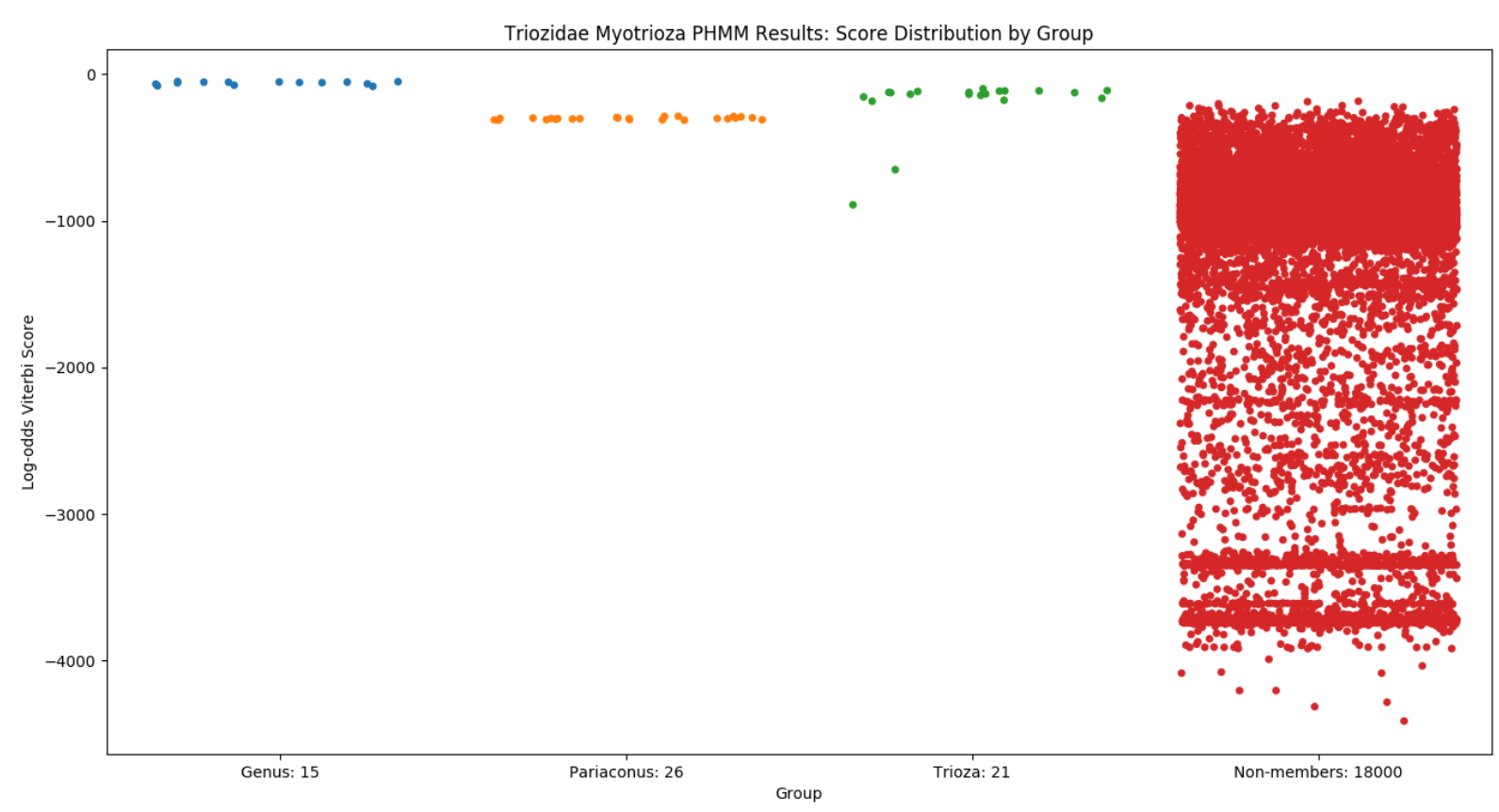

Fig. 25. Results from a profile hidden Markov model trained on Triozidae Myotrioza.

TABLE XXVI

TRIOZIDAE MYOTRIOZA RESULTS SUMMARY

\begin{tabular}{|c|c|c|c|c|c|}
\hline \multirow[b]{2}{*}{ Testing } & \multicolumn{5}{|c|}{ Triozidae Myotrioza (РНMМ) } \\
\hline & $\begin{array}{c}\text { Minimum score } \\
\text { (threshold for self) }\end{array}$ & $\begin{array}{l}\text { Maximum } \\
\text { score }\end{array}$ & Size & $\begin{array}{c}\text { \# Classified } \\
\text { correctly }\end{array}$ & Accuracy \\
\hline Myotrioza (self) & -84.5239 & -40.7887 & 15 & 15 & $100 \%$ \\
\hline Pariaconus & -315.1446 & -285.5164 & 26 & 26 & $100 \%$ \\
\hline Trioza & -892.0864 & -98.7706 & 21 & 21 & $100 \%$ \\
\hline Non-members & -4410.6429 & -169.2690 & 180000 & 180000 & $100 \%$ \\
\hline
\end{tabular}




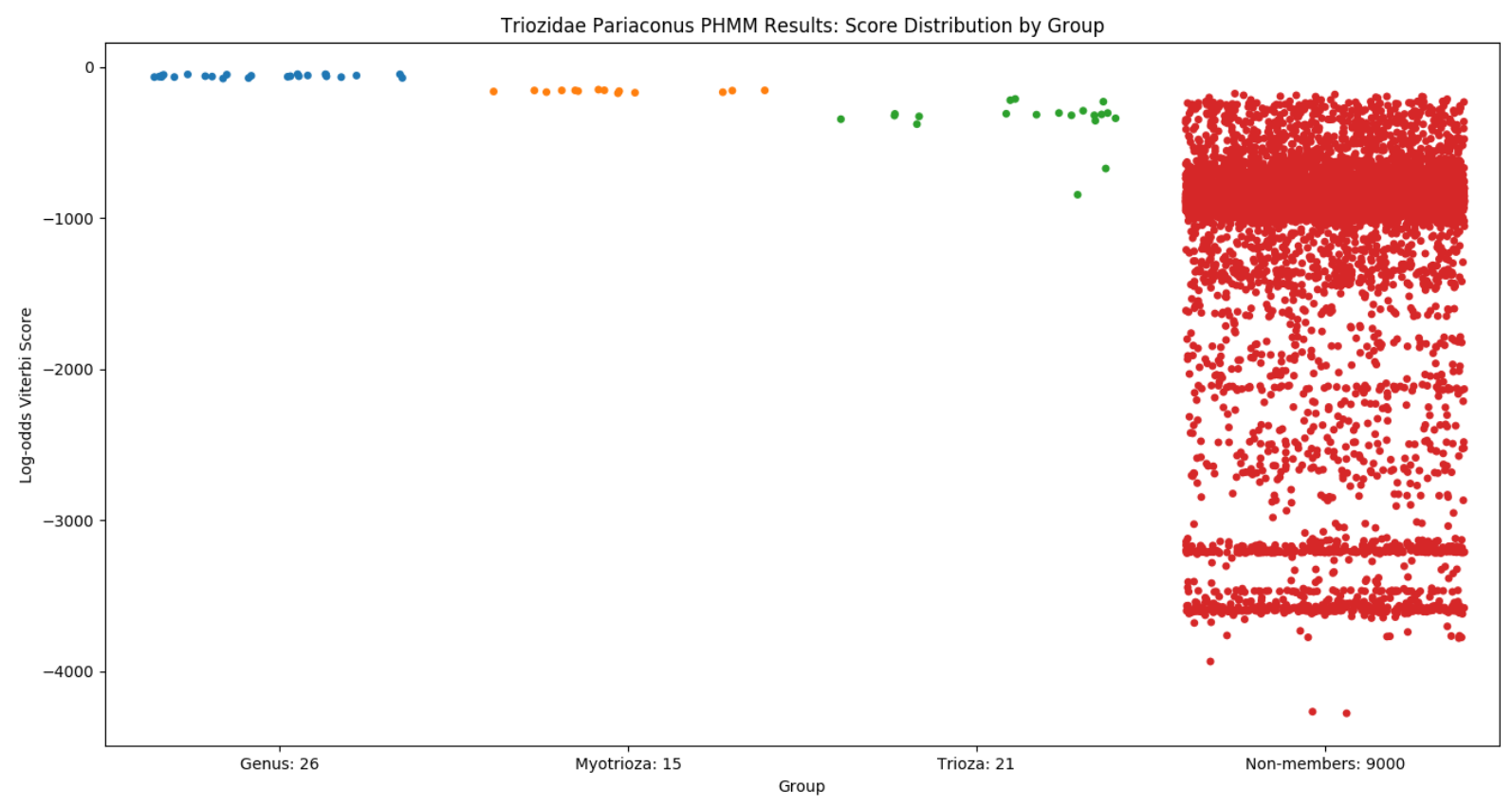

Fig. 26. Results from a profile hidden Markov model trained on Triozidae Pariaconus.

TABLE XXVII

TRIOZIDAE PARIACONUS RESULTS SUMMARY

\begin{tabular}{|c|c|c|c|c|c|}
\hline \multirow[b]{2}{*}{ Testing } & \multicolumn{5}{|c|}{ Triozidae Pariaconus (PHMM) } \\
\hline & $\begin{array}{l}\text { Minimum score } \\
\text { (threshold for self) }\end{array}$ & $\begin{array}{l}\text { Maximum } \\
\text { score }\end{array}$ & Size & \# Classified correctly & Accuracy \\
\hline Pariaconus (self) & -76.9430 & -47.9023 & 26 & 26 & $100 \%$ \\
\hline Myotrioza & -174.3121 & -149.7725 & 15 & 15 & $100 \%$ \\
\hline Trioza & -845.7142 & -121.2870 & 21 & 21 & $100 \%$ \\
\hline Non-members & -4277.3265 & -167.2943 & 9000 & 9000 & $100 \%$ \\
\hline
\end{tabular}




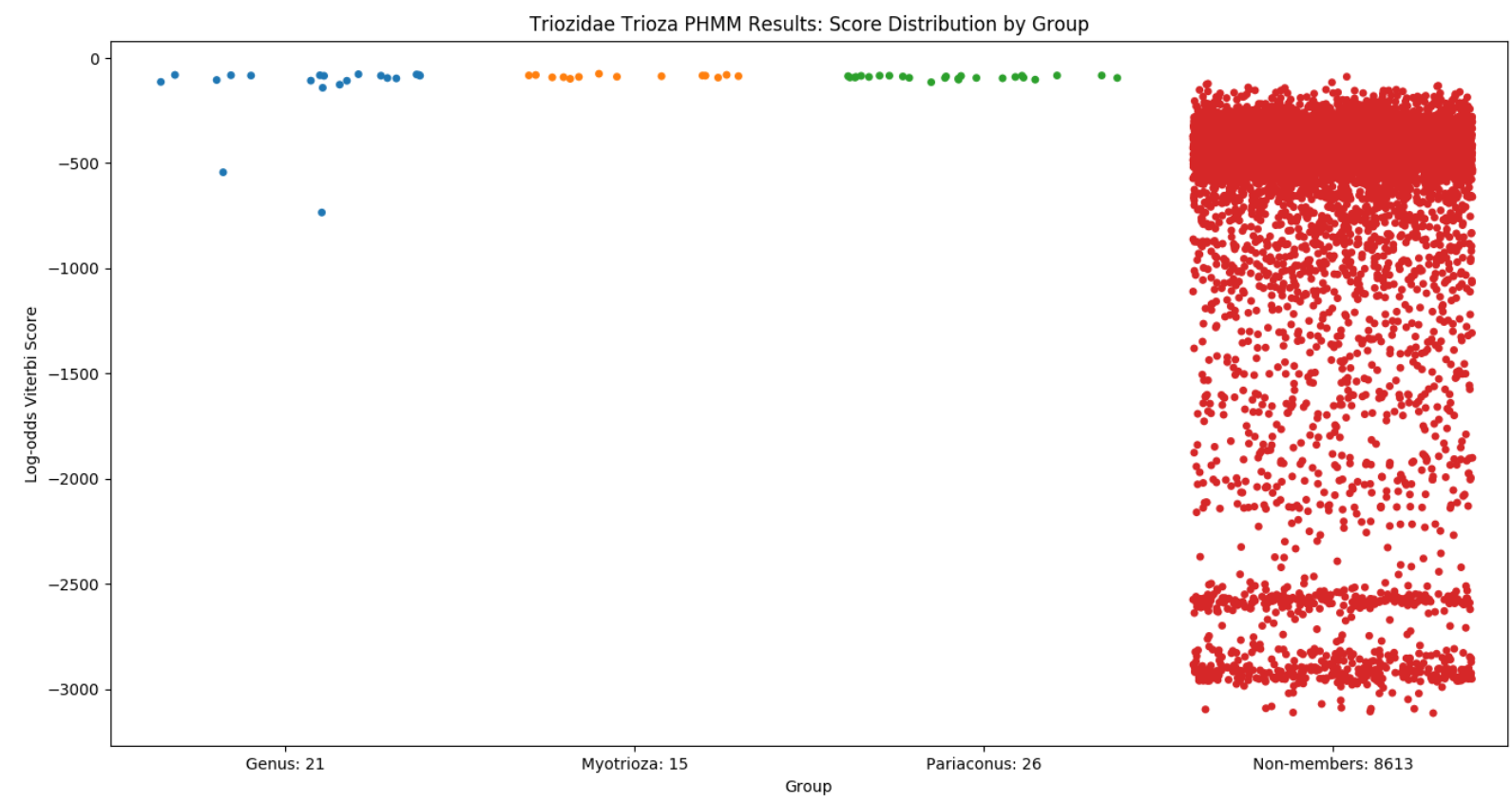

Fig. 27. Results from a profile hidden Markov model trained on Triozidae Trioza.

TABLE XXVIII

TRIOZIDAE TRIOZA RESULTS SUMMARY

\begin{tabular}{|c|c|c|c|c|c|}
\hline \multirow[b]{2}{*}{ Testing } & \multicolumn{5}{|c|}{ Triozidae Trioza (PHMM) } \\
\hline & $\begin{array}{c}\text { Minimum score } \\
\text { (threshold for self) }\end{array}$ & $\begin{array}{l}\text { Maximum } \\
\text { score }\end{array}$ & Size & $\begin{array}{l}\text { \# Classified } \\
\text { correctly }\end{array}$ & Accuracy \\
\hline Trioza (self) & $-141.5907^{*}$ & -75.2848 & 21 & 19 & $90.48 \%$ \\
\hline Myotrioza & -99.1209 & -71.7936 & 15 & 0 & $0 \%$ \\
\hline Pariaconus & -115.3349 & -80.1063 & 26 & 0 & $0 \%$ \\
\hline Non-members & -3113.2447 & -83.7276 & 8613 & 8605 & $99.91 \%$ \\
\hline
\end{tabular}

*Disqualified scores: $-734.3832,-543.3373$. 


\section{Vespinae}

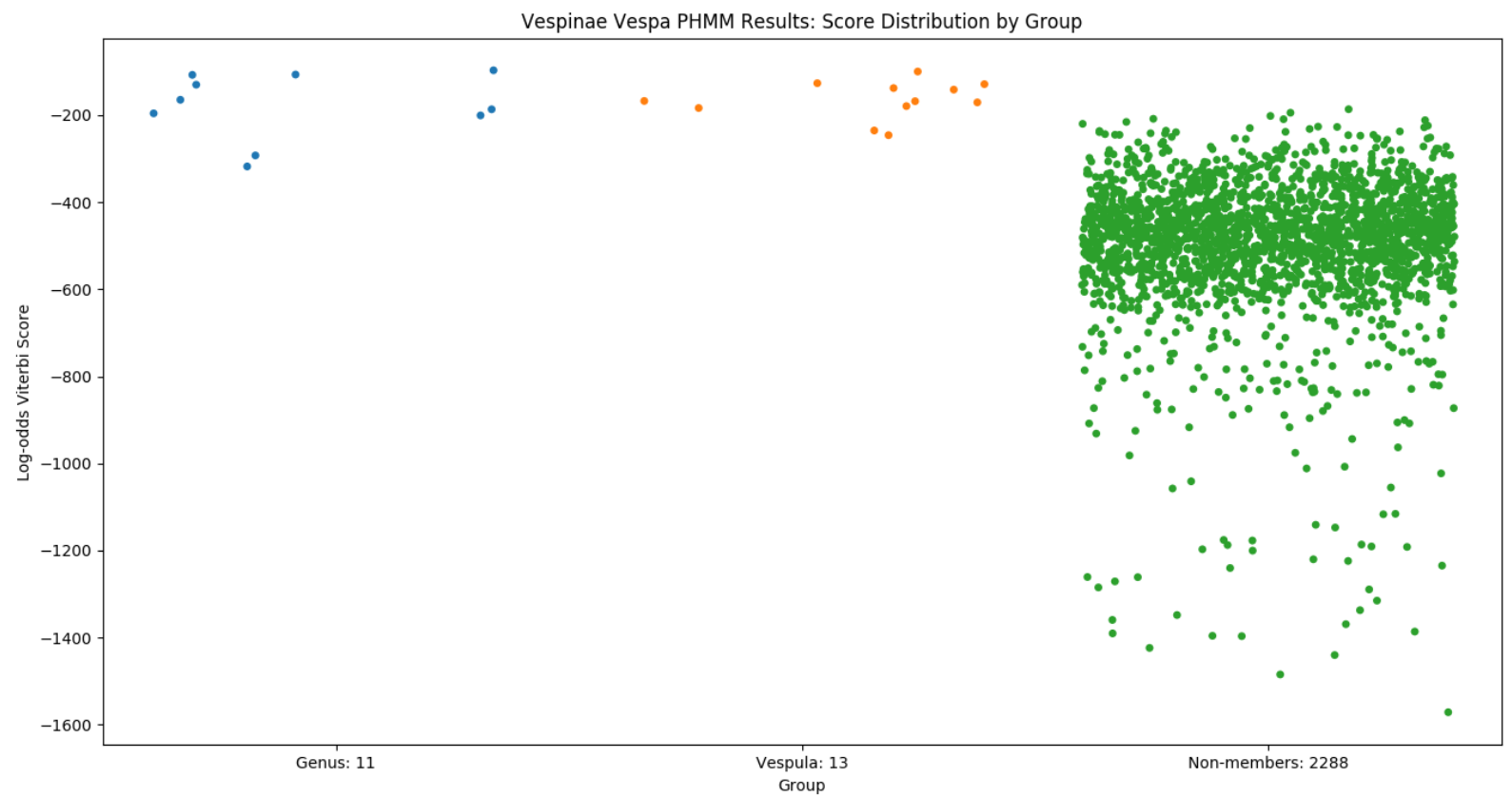

Fig. 28. Results from a profile hidden Markov model trained on Vespinae Vespa.

TABLE XXIX

VESPINAE VESPA RESULTS SUMMARY

\begin{tabular}{|c|c|c|c|c|c|}
\hline \multirow[b]{2}{*}{ Testing } & \multicolumn{5}{|c|}{ Vespinae Vespa (РНMM) } \\
\hline & $\begin{array}{c}\text { Minimum score } \\
\text { (threshold for self) }\end{array}$ & $\begin{array}{l}\text { Maximum } \\
\text { score }\end{array}$ & Size & $\begin{array}{l}\text { \# Classified } \\
\text { correctly }\end{array}$ & Accuracy \\
\hline Vespa (self) & -317.9715 & -84.4424 & 11 & 11 & $100 \%$ \\
\hline Vespula & -246.1331 & -98.8176 & 13 & 0 & $0 \%$ \\
\hline Non-members & -1571.5790 & -156.2421 & 2288 & 2159 & $94.36 \%$ \\
\hline
\end{tabular}


Vespinae Vespula PHMM Results: Score Distribution by Group

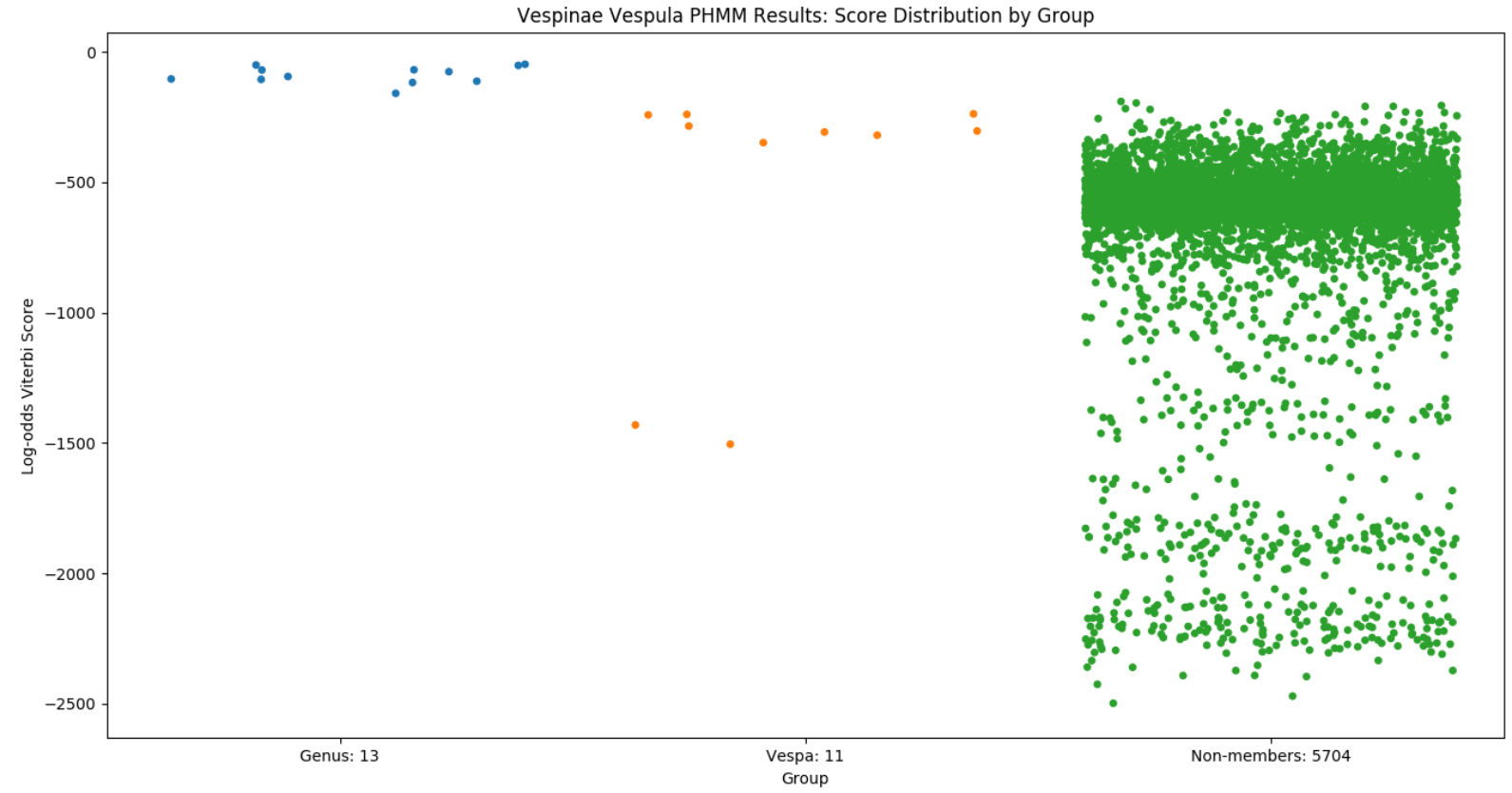

Fig. 29. Results from a profile hidden Markov model trained on Vespinae Vespula.

TABLE XXX

VESPINAE VESPULA RESULTS SUMMARY

\begin{tabular}{|c|c|c|c|c|c|}
\hline \multirow{2}{*}{ Testing } & \multicolumn{4}{|c|}{ Vespinae Vespula (PHMM) } \\
\cline { 2 - 6 } & $\begin{array}{c}\text { Minimum score } \\
\text { (threshold for self) }\end{array}$ & $\begin{array}{c}\text { Maximum } \\
\text { score }\end{array}$ & Size & \# Classified correctly & Accuracy \\
\hline Vespula (self) & -158.9880 & -44.6717 & 13 & 13 & $100 \%$ \\
\hline Vespa & -1504.2608 & -115.9735 & 11 & 10 & $90.91 \%$ \\
\hline Non-members & -2497.7260 & -147.4746 & 5704 & 5703 & $99.98 \%$ \\
\hline
\end{tabular}




\section{Precision and Recall}

Table XXXI shows the precision and recall for each genus PHMM.

Each metric was calculated in relation to the genus and is described below:

- True positives: The number of genus members that were correctly classified as members of the genus

- False negatives: The number of genus members that were incorrectly classified as non-members of the genus

- False positives: The number of family members and non-members that were incorrectly classified as members of the genus

- True negatives: The number of family members and non-members that were correctly classified as non-members of the genus

- Precision: The percentage of members that were reported as genus members, that are actually members of the genus

- Recall: The percentage of genus members from the original dataset, that were reported as genus members (also known as the true positive rate or sensitivity) 
TABLE XXXI

PRECISION AND RECALL FOR GENUS CLASSIFICATION

\begin{tabular}{|c|c|c|}
\hline Family Genus & Precision & Recall \\
\hline Acanthosomatidae Acanthosoma & $60.00 \%$ & $100 \%$ \\
\hline Acanthosomatidae Elasmucha & $19.40 \%$ & $100 \%$ \\
\hline Agabini Agabus & $64.86 \%$ & $92.31 \%$ \\
\hline Agabini Ilybius & $37.25 \%$ & $100 \%$ \\
\hline Agabini Platynectes & $31.15 \%$ & $100 \%$ \\
\hline Agelenidae Malthonica & $50 \%$ & $100 \%$ \\
\hline Agelenidae Sinocoelotes & $53.57 \%$ & $100 \%$ \\
\hline Agelenidae Tegenaria & $9.71 \%$ & $100 \%$ \\
\hline Harpalini Harpalus & $90 \%$ & $96.43 \%$ \\
\hline Harpalini Ophonus & $91.67 \%$ & $100 \%$ \\
\hline Hydraenidae Hydraena & $6.83 \%$ & $100 \%$ \\
\hline Hydraenidae Limnebius & $83.67 \%$ & $78.85 \%$ \\
\hline Hydraenidae Ochthebius & $63.93 \%$ & $98.73 \%$ \\
\hline Hydropsychinae Ceratopsyche & $20.65 \%$ & $95.00 \%$ \\
\hline Hydropsychinae Cheumatopsyche & $41.25 \%$ & $91.67 \%$ \\
\hline Hydropsychinae Hydromanicus & $17.33 \%$ & $100 \%$ \\
\hline Hydropsychinae Hydropsyche & $52.29 \%$ & $96.61 \%$ \\
\hline Hydropsychinae Orthopsyche & $19.75 \%$ & $100 \%$ \\
\hline Hydropsychinae Potamyia & $52.00 \%$ & $100 \%$ \\
\hline Ophiolepididae Ophiolepis & $3.37 \%$ & $100 \%$ \\
\hline Ophiolepididae Ophiomusium & $2.09 \%$ & $100 \%$ \\
\hline Triozidae Myotrioza & $100 \%$ & $100 \%$ \\
\hline Triozidae Pariaconus & $100 \%$ & $100 \%$ \\
\hline Triozidae Trioza & $27.94 \%$ & $90.48 \%$ \\
\hline Vespinae Vespa & $7.19 \%$ & $100 \%$ \\
\hline Vespinae Vespula & $86.67 \%$ & $100 \%$ \\
\hline
\end{tabular}




\section{Chapter 6: Discussion}

This chapter discusses the overall performance regarding each of the three evaluation options, analyzes possible effects of genus size and sequence length on our model accuracies, describes challenges we encountered in this project, and outlines steps for future expansion.

\section{Overall Performance}

\section{Testing on Non-Members}

Out of the 26 genera PHMMs in this experiment, 24 received over 94\% accuracy when tested on non-members, and 22 of these 24 received over $99 \%$ accuracy. The remaining lower-performing genera, Ophiolepis and Ophiomusium, had non-member evaluation accuracies of $84.02 \%$ and $73.88 \%$. In addition, these genera were both members of and the only members evaluated from the family Ophiolepididae.

The results of this project indicate that PHMMs can clearly differentiate the majority of non-members between genus members.

\section{Testing on Family Members}

Acanthosomatidae Acanthosoma, Acanthosomatidae Elasmucha, Agabini Ilybius, Agabini Platynectes, Harpalini Harpalus, Harpalini Ophonus, Hydraenidae Ochthebius, Hydropsychinae Ceratopsyche, Hydropsychinae Hydromanicus, Triozidae Myotrioza, 
Triozidae Pariaconus, and Vespinae Vespula generally showed the most ideal relationship between genus, family, and non-members. In each, the genus members clearly scored the highest. The two other family member genera scored lower than the genus but generally not as low as non-members. Additionally, each individual family member genus scored in a predictable range; the plots for them were almost constant lines. Given an unknown sequence that we suspect to be in the family, we could make predictions on which genus in the family it belongs to through these genus PHMMs alone.

However, this was not the majority case. For example, in the Agelenidae Malthonica and Agelenidae Tegenaria PHMMs, family member Sinocoelotes scored extremely low; lower than the majority of non-members. This is likely due to the length of Sinocoelotes sequences: all ranged from 1037 to 1194 bp, whereas Malthonica sequences were all 471 bp and Tegenaria sequence lengths ranged from 325 to $1194 \mathrm{bp}$.

Other PHMMs, such as Hydropsychinae Hydropsyche and Ophiolepididae Ophiomusium, showed confusion between family and genus members. Numerous family members scored higher than the genus; therefore, if only using the minimum threshold for the genus, all of these family members would be false positives. Therefore, to increase the accuracy for the model classification, we could calculate the maximum threshold from the maximum score from the genus members and only classify sequences that score within the range between the two thresholds, as possible members of the genus. 
While we would like to see a clearer separation between family members in the ideal case, PHMM confusion is not surprising - this may just indicate that family member sequences are similar to each other.

\section{Testing on Genus Members}

We are interested in knowing whether or not this method could help us determine whether or not an unknown sequence, suspected to be from a genus, can be classified as a member of that genus based on the threshold determined from this project.

For this, we can look at the the "self" accuracy column in the genus tables from the previous section or the "recall" column in Table XXXI; these represent the same values. We can disregard the 18 genera with $100 \%$ recall; these thresholds were simply chosen as the minimum score produced by the dataset. Instead, we can observe the adjusted threshold scores (marked with asterisks in the genus tables). Because these adjustments will always make the threshold closer to 0 , they decrease the range of possible scores to be classified as a genus member and will therefore always guarantee an improvement in accuracy when testing family members or non-members against the genus PHMM. In other words, it will always decrease the number of false positives but will conversely increase the number of false negatives (genus members that are not correctly classified as genus members).

Knowing this, we would like to additionally observe whether or not adjusting thresholds in some genera by "disqualifying" outlier low scores still resulted in a high accuracy if used to classify its own members. Therefore, we would like to minimize the number of false 
negatives and loss of information if we were to disregard these scores. The ideal adjusted threshold would not only raise the accuracy when testing family and non-members (guaranteed), but also still give us a high recall by classifying genus members correctly (not guaranteed). In this project, we have considered any recall of $90 \%$ or above after disqualifying outliers to be "accurate."

For example, for Agabini Agabus, we disqualified the scores -526.6488 and -448.7219 from being chosen to use for the threshold. The next lowest score, -144.1835 , was chosen. This resulted in 24 Agabus members being classified correctly as an Agabus member and the disqualified 2 being classified incorrectly, giving us a recall of 92.31\%. Since we are only disqualifying 2 sequences, we consider this as a fairly low loss of information for now, until the acquired dataset for this genus becomes more diverse.

Out of the 8 adjusted thresholds (from Agabini Agabus, Harpalini Harpalus, Hydraenidae Limnebius, Hydraenidae Ochthebius, Hydropsychinae Ceratopsyche, Hydropsychinae Cheumatopsyche, Hydropsychinae Hydropsyche, and Triozidae Trioza), 7 retained an recall of greater than 90\%. Only Hydraenidae Limnebius had a lower recall: after disqualifying scores that ranged from -273.2947 to -324.1376 due to the large difference from the majority of the other scores, we selected the next lowest score, -160.0001 . This improved our Hydraena (family member) accuracy by 85\%, our Ochthebius (family member) accuracy by $96 \%$, and and our non-member accuracy by $5 \%$. However, only 41 out of 52 genus sequences were classified correctly, resulting in a recall of 78.85\%. Evidently, although this adjustment improved the family member and non-member accuracy, it did 
not retain an acceptable genus accuracy and is therefore not a threshold that is representative enough of the genus members. Different methods of threshold calculation can be further researched in the future.

To assess the success of our genus identification, we are additionally interested in the "precision" column of Table XXXI. High precision indicates that the genus model reported a low number of false positives (non-genus-members incorrectly classified as genus members) and a high number of true positives (genus members correctly classified as genus members). If we were to present an unknown sequence to a model with high precision, we could trust that the sequence was a member of a particular genus if the model reported it as such. Otherwise, if the model had low precision, it could incorrectly report the sequence as a genus member.

Unfortunately, only 4 out of 26 genera had a precision of approximately $90 \%$ or higher. Of these, only Triozidae Myotrioza and Triozidae Pariaconus received a precision and recall of $100 \%$. Out of the remaining 20 genera, 2 achieved a precision above $80 \%$. The remaining 18 genera all had precision that was under $65 \%$. This was in contrast to the genera's high recall. This indicates that, while the genus PHMMs were able to mostly classify all genus members as genus members, they also misclassified family members or non-members as genus members by using the same threshold. While low precision is undesirable, it is not an immediate indication that PHMMs are inadequate at genus classification. Instead, the low precision may be due to the differing number of sequences used to test each genus; if each PHMM was tested on all sequences in the initial dataset, the precision of each PHMM 
would be more comparable and representative of this method's potential. Another possibility is that the success of the PHMMs were affected by the features of our input data that was used to build each training set, and that we must first thoroughly pre-process the data before building the PHMMs. In the next subsection, we will describe possible correlations between our results and these factors, as well as possible solutions.

\section{Accuracy Correlation Analysis}

Originally, we had expected that accuracy would have a positive correlation with genus size. However, the results from this project did not show a consistent relationship between these two metrics. In fact, genus size appeared to have a negative effect on accuracy. For example, for the Hydraenidae Hydraena PHMM, Limnebius scored $15.38 \%$ accuracy and Ochthebius scored a $1.27 \%$ accuracy - extremely low despite the large genus sizes $(88,52$, and 79 , respectively).

At first, this was disconcerting. As we expect to acquire much more data in the future and therefore increase genus sizes in our dataset, assessing this experiment by only the relationship between genus size and accuracy would make one believe that acquiring more data would render this approach useless. However, this led me to look further into the effects of larger genus size, namely, the sequence length in the members of the genera. Larger genera tended to have a much broader range of sequence lengths, which may have made the PHMM less precise in differentiating between genus, family, and non-members. 
One of the goals of Heller et al.'s CO-ARBitrator was to acquire COI sequences from GenBank that may have not been included in the BOLD database due to its lengthy acceptance process and requirements [13]. This was successful in that CO-ARBitrator was able to acquire 507,651 COI sequences from GenBank that were not in BOLD. However, because BOLD requires that the sequences submitted to their database for consideration are trimmed by trusted primers [11], sequences from BOLD tend to be more uniform. Since BOLD also uses a COI hidden Markov model to test sequences in its approval process, it makes sense that they require sequences to be isolated by primers. If not, the sequences might score poorly despite being the same organism or from the same genus, as we have shown in our experiment.

A possible solution to this that would allow us to still utilize the larger CO-ARBitrator is to implement software with a COI primer database that trims CO-ARBitrator with trusted forward and reverse primers. The next subsection will describe our attempt at this method.

\section{Primer Isolation Method}

We acquired a set of 48 known forward COI primers and a set of 19 known reverse COI primers. Because these primers contained IUPAC nucleotide code ${ }^{3}$ to represent multiple

\footnotetext{
${ }^{3}$ https://www.bioinformatics.org/sms/iupac.html
} 
possible nucleotides in one position, we referenced the IUPAC translation table and created regular expressions to represent each individual primer.

Then, for each sequence in the training set before creating a PHMM, the program checked whether it contained any of the forward primer regular expressions, and returned the index of the first match found. If found, the program trimmed the sequence from the beginning of the sequence to the end of the forward primer. It then similarly searched for a reverse primer. If found, the program trimmed the sequence from the start of the reverse primer until the end of the sequence.

While this method is promising, it did not improve our results. This is because one forward primer was found in most sequences, but there was no reverse primer with similar success. Despite including the corresponding reverse primer for the successful forward primer, this reverse primer was not found in most sequences. We attribute this to needing more trusted primers in our dataset. In the future, all primers from BOLD's primer database ${ }^{4}$ could be tried.

${ }^{4}$ http://v3.boldsystems.org/index.php/Public_Primer_PrimerSearch 


\section{Machine Limitations}

It takes approximately one minute to calculate Viterbi log-odds score for one sequence using Dr. Philip Heller's ProfileHMM class. This work was parallelized to be executed by the HPC. With 23 cores for each job submitted, we had expected that the HPC would be able to complete testing 9,000 non-members in approximately five hours for one genus.

Unfortunately, this was only the case for two genera. In the majority of cases, the execution times of jobs on the HPC varied and were faced with obstacles, of which the solutions were beyond our control.

For example, the execution time could sometimes largely depend on the number or size of jobs submitted by all users with access to the HPC. In addition, there was a factor of randomness. Some nodes that were assigned jobs were destined to be particularly successful, and some nodes were more prone to failure. For example, as mentioned above, two nodes to which our project's jobs were assigned, performed extremely quickly and completed testing 9,000 non-members in approximately five hours. However, some nodes would only finish testing 150 non-members in the same amount of time.

Furthermore, nodes on the HPC would sometimes shut down and without warning. The job would execute from the beginning as if it were being executed for the first time, and it would overwrite all output and progress that had been made. This instability was inconsistent; it was sometimes caused by a large total number of jobs currently being executed but would sometimes occur without an explainable reason. 
Although this project was also executed on two personal machines, the personal machines could not produce sizeable data like the HPC; therefore, most of the family member and genus tests were performed on the personal machines, and all non-member tests were performed on the HPC.

Because of these reasons, we were not able to test this experiment on more families and genera, nor were we able to complete testing all 9,000 sequences on each genus included in this project.

\section{Future Directions}

In this section we have described ideas for future projects.

\section{Preprocessing Deflines from Input Data}

This subproject involves formatting the deflines of the original input file so that only the main taxonomic ranks (kingdom, phylum, class, order, family, and genus) are retained. This would change our tree to be a balanced tree. Because our taxonomic tree is currently unbalanced, we attempt to determine genus nodes as those that are " 2 levels from the species (leaf) nodes," phylum nodes as those that are "1 level from the kingdom (root) node," and so on. However, this is problematic. In the example in Fig. 1, the Anthocoridae node for Orius sauteri exists 13 levels from the root and 3 levels from a leaf. In contrast, Anthocoridae for Orius nagaii exists 4 levels from the root and 2 levels from a leaf. Because of these differing distances from both the root and the leaves, these nodes would not be 
able to be accessed together using these measures alone. This would therefore lead to a loss of information: these nodes represent the same taxonomic rank with the same group name, but the program cannot access them together and therefore cannot combine their children. This issue similarly arises in the situations where taxonomic groups are misannotated, or where one taxonomic group is represented under a variation of group names. An alternative solution would be to traverse the entire tree and combine nodes with any shared group names, but this would be extremely inefficient and undesirable.

In addition, in our project, the class Gastropoda was one of the nodes that was two levels above the species nodes. We would expect that class nodes would only be in levels that are three levels above species or higher, since class is three levels above species in taxonomic ranking. Since Gastropoda is a class and not a family, the number of sequences was hugely disproportionate to the other nodes and it was discarded from the qualifying families. If all deflines were uniform, we would not need to perform this additional inspection.

With a balanced tree, nodes could be compared to only nodes also at the same taxonomic rank, instead of comparing "nodes that are two levels from the species (leaf) nodes" that may still consist of different taxonomic ranks (in our project: family, tribe, subfamily, and so on). In addition, each taxonomic group name could be represented by a single node instead of being represented at different levels. This data preprocessing would therefore allow us to more accurately compare performance of PHMMs made at certain taxonomic ranks, while maximizing the amount of data provided by each node. 


\section{Handling Varying Sequence Length and Disqualifying Outliers}

We have described the primer isolation method as a strategy to handle varying sequence length. Only after isolating all COI sequences using primers, the program can create PHMMs at the genus level to determine the threshold score. If there are still outliers in the genus despite having trimmed the sequences with primers, these outlier sequences can be discarded from the training set. Then, the program can once again create PHMMs at the genus level. It would be interesting to find whether or not primer isolation by itself does improve PHMM accuracy and reduce the number of outliers in each genus.

\section{Creating Multiple PHMMs for Each Genus}

If there are genus PHMMs that still do not improve after primer isolation and outlier disqualification, low accuracy may be due to still consisting of an even ratio of short and long sequences. For example, if a genus has twelve 400-bp sequences and twelve 1000-bp sequences, it may be more effective to create separate PHMMs for this genus: one trained on the 400-bp sequences and one trained on the 1000-bp sequences. Given an unknown sequence, if we suspect that it is from this genus, we can test it against both genus PHMMs. If there is a significant hit to either of these PHMMs over the scores from other PHMMs, we can have confidence that our unknown sequence might be from this genus.

The disadvantage to this method is that it would require much more time and computing power, since multiple PHMMs are being created and trained. However, it might reduce the 
number of false negatives and false positives because the resulting threshold would be more representative of sequences of a particular length range in a genus.

\section{Further Analyzing Sequence Length vs. PHMM Score}

To further study the relationship between sequence length and PHMM score, in the future we could plot graphs that plot the log-odds score in relation to the sequence length.

\section{Testing on Entire Input Data}

Due to machine limitations, we were unable to test the PHMMs on the entire input dataset. With more computing power and time in the future, it would be valuable to test on all original input sequences to gather more information on accuracy and performance. 


\section{References}

[1] E. Mayr, Growth of Biological Thought: Diversity, Evolution, and Inheritance, vol. 11, Cambridge, Massachusetts: The Belknap Press of Harvard University, 1982, pp. 147297.

[2] F. L. Mantelatto et al., "DNA sequence database as a tool to identify decapod crustaceans on the São Paulo coastline," Mitochondrial DNA Part A, pp. 1-11, 5 Sept. 2017.

[3] J. B. Geller, "Decline of a native mussel masked by sibling species invasion," Conservation Biology, vol. 13, no. 3, pp. 661-664, June 1999.

[4] A. Valentini, F. Pompanon, and P. Taberlet, "DNA barcoding for ecologists," Trends in Ecology \& Evolution, vol. 24, no. 2, pp. 110-117, Feb. 2009.

[5] R. B. Franklin and A. L. Mills, "Multi-scale variation in spatial heterogeneity for microbial community structure in an eastern Virginia agricultural field," FEMS Microbiology Ecology, vol. 44, no. 3, pp. 335-346, June 2003.

[6] P. D. N. Hebert and T. R. Gregory, "The promise of DNA barcoding for taxonomy," Systematic Biology, vol. 54, no. 1, pp. 852-859, Oct. 2005.

[7] P. D. N. Hebert, E. H. Penton, J. M. Burns, D. H. Janzen, and W. Hallwachs, "Ten species in one: DNA barcoding reveals cryptic species in the neotropical skipper butterfly 
Astraptes fulgerator," Proceedings of the National Academy of the United States of America, vol. 101, no. 41, pp. 14812-14817, 12 Oct. 2004.

[8] A. Galimberti et al., "DNA barcoding as a new tool for food traceability," Food Research International, vol. 50, no. 1, pp. 55-63, Jan. 2013.

[9] P. D. N. Hebert, A. Cywinska, S. L. Ball, and J. R. deWaard, "Biological identifications through DNA barcodes," Proceedings: Biological Sciences, vol. 270, no. 1512, pp. 313321, Feb. 2003.

[10] E. D. Stein, M. C. Martinez, S. Stiles, P. E. Miller, and E. V. Zakharov, "Is DNA barcoding actually cheaper and faster than traditional morphological methods: results from a survey of freshwater bioassessment efforts in the United States?," PLoS One, vol. 9, no. 4, pp. 1-10, Apr. 2014.

[11] S. Ratnasingham and P. D. N. Hebert, "BOLD: The Barcode of Life Data System," Molecular Ecology Notes, vol. 7, pp. 355-364, 2007.

[12] M. Leray, S.-L. Ho, I.-J. Lin, and R. J. Machida, "MIDORI server: a webserver for taxonomic assignment of unknown metazoan mitochondrial-encoded sequences using a curated database," Bioinformatics, vol. 34, no. 21, pp. 3753-3754, June 2018. 
[13] P. Heller, J. Casaletto, G. Ruiz, and J. Geller, "A database of metazoan cytochrome c oxidase subunit I gene sequences derived from GenBank with CO-ARBitrator," Scientific Data, vol. 5, pp. 1-7, Aug. 2018.

[14] H. J. Tripp, I. Hewson, S. Boyarsky, J. M. Stuart, and J. P. Zehr, "Misannotations of rRNA can now generate $90 \%$ false positive protein matches in metatranscriptomic studies," Nucleic Acids Research, vol. 39, no. 20, pp. 8792-8802, June 2011.

[15] P. Heller, H. J. Tripp, K. Turk-Kubo, and J. P. Zehr, "ARBitrator: a software pipeline for on-demand retrieval of auto-curated nifH sequences from GenBank," Bioinformatics, vol. 30, no. 20, pp. 2883-2890, July 2014.

[16] S. F. Altschul, W. Gish, W. Miller, E. W. Myers, and D. J. Lipman, "Basic local alignment search tool," Journal of Molecular Biology, vol. 215, no. 3, pp. 403-410, Oct. 1990.

[17] V. Sharma, "Genetic barcode identification with profile hidden Markov models," San José State University, San José, 2018. 